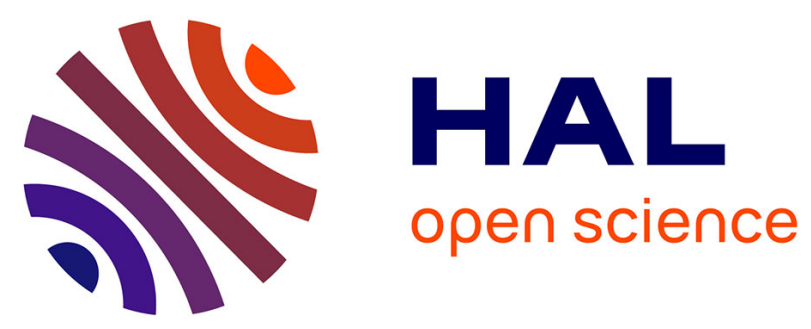

\title{
Approximate local Dirichlet-to-Neumann map for three-dimensional time-harmonic elastic waves
}

Stéphanie Chaillat, Marion Darbas, Frédérique Le Louër

\section{To cite this version:}

Stéphanie Chaillat, Marion Darbas, Frédérique Le Louër. Approximate local Dirichlet-to-Neumann map for three-dimensional time-harmonic elastic waves. Computer Methods in Applied Mechanics and Engineering, 2015, 297 (1), pp.62-83. 10.1016/j.cma.2015.08.013 . hal-01187242

\section{HAL Id: hal-01187242 \\ https://hal.science/hal-01187242}

Submitted on 27 Aug 2015

HAL is a multi-disciplinary open access archive for the deposit and dissemination of scientific research documents, whether they are published or not. The documents may come from teaching and research institutions in France or abroad, or from public or private research centers.
L'archive ouverte pluridisciplinaire HAL, est destinée au dépôt et à la diffusion de documents scientifiques de niveau recherche, publiés ou non, émanant des établissements d'enseignement et de recherche français ou étrangers, des laboratoires publics ou privés. 


\title{
Approximate local Dirichlet-to-Neumann map for three-dimensional time-harmonic elastic waves.
}

\author{
Stéphanie Chaillat ${ }^{\mathrm{a}}$, Marion Darbas ${ }^{\mathrm{b}, *}$, Frédérique Le Louërc \\ ${ }^{a}$ Laboratoire POems UMR 7231 CNRS-INRIA-ENSTA, \\ ENSTA-UMA, 828 Bd des Maréchaux, 91762 Palaiseau Cedex, FRANCE \\ ${ }^{b}$ LAMFA UMR CNRS 7352 \\ Université de Picardie Jules Verne, 33 rue Saint-Leu 80039 Amiens Cedex, FRANCE \\ ${ }^{c} L M A C E A$ 2222 \\ Université de Technologie de Compiègne, 60205 Compiègne Cedex, FRANCE
}

\begin{abstract}
It has been proven that the knowledge of an accurate approximation of the Dirichlet-to-Neumann $(\mathrm{DtN})$ map is useful for a large range of applications in wave scattering problems. We are concerned in this paper with the construction of an approximate local DtN operator for time-harmonic elastic waves. The main contributions are the following. First, we derive exact operators using Fourier analysis in the case of an elastic half-space. These results are then extended to a general three-dimensional smooth closed surface by using a local tangent plane approximation. Next, a regularization step improves the accuracy of the approximate DtN operators and a localization process is proposed. Finally, a first application is presented in the context of the On-Surface Radiation Conditions method. The efficiency of the approach is investigated for various obstacle geometries at high frequencies.
\end{abstract}

Keywords: Scattering, time-harmonic elastic waves, approximate local DtN map, far-field patterns

\section{Introduction}

The simulation of time-harmonic elastic waves in the high frequency regime is a timely research field due to the variety of possible applications (for example seismology, remote sensing or nondestructive testing). To solve elastodynamic scattering problems in unbounded domains, various numerical methods are used [56]. We can mention the Finite Element Method [41, 25, 43, 13, or the Finite Difference Method [55, 38]. Such volume methods are used with nonreflecting boundary conditions [37, 35] such as Absorbing Boundary Conditions (ABCs) [27, 20, 34] or Perfectly Matched Layers (PMLs) [12, 40, 26] to truncate the computational domain. Another possible approach is to use the Fast Multipole accelerated Boundary Element Method (FM-BEM) [22, 23, 21] resulting from the discretization of Boundary Integral Equations (BIE) 47]. Some of the above mentioned numerical methods reveal to be unstable or ill-conditioned in the high frequency regime. Overcoming this problem requires the knowledge of a good approximation of the Dirichlet-to-Neumann map (DtN). The aim of this paper is to propose local approximations of the DtN map for 3D frequency-domain elastodynamics in the high frequency regime. The derivation process falls into the techniques of the On-Surface Radiation Conditions (OSRC) method [44, 3, 7, 33. It is worth noting that approximate DtN operators are not limited to the OSRC method but find many applications. They are used to derive absorbing boundary conditions for infinite domains. We can

\footnotetext{
* Corresponding author

Email addresses: stephanie.chaillat@ensta-paristech.fr (Stéphanie Chaillat), marion.darbas@u-picardie.fr (Marion Darbas), frederique.le-louer@utc.fr (Frédérique Le Louër)
} 
cite for example [11, 42, 4, 9, 10, 51] in acoustics and electromagnetism and [37, 39, 35, in elastodynamics. These approximations play also a crucial role in Domain Decomposition Methods (DDM) 36, 14 to ensure the transmission of the physical informations between the sub-domains. In the DDM context, algebraic approximations of the DtN have been proposed [49, 50]. Furthermore, approximate local DtN are very useful to derive fast iterative solutions of scattering problems by the integral equations method. Since fine meshes leading to large-scale systems are used in the high frequency regime, the application of direct methods is prohibitive (due to large CPU times and memory requirements). An alternative is to consider iterative solvers. But linear systems arising from the discretization of the elastodynamic equations are known to be difficult to solve at high wavenumbers since the matrix tends to be indefinite. For example, it has been shown that the number of iterations can significantly hinder the overall efficiency of the FMBEM [22, 23]. Traditional algebraic preconditioning approaches such as incomplete LU, SParse Approximative Inverse [18, 19], multi-grid methods [17], nested GMRES algorithm [24] have been applied to electromagnetic or elastodynamic FM-BEMs. However, since algebraic preconditioners use only a small part of the system matrix, they do not contain enough information on the physics of the underlying continuous operator and cannot always improve the performance of the solver for high frequencies. Analytical preconditioners offer a very interesting alternative. They play the role of regularizing operators in the integral representation of the scattered field and improve the spectral properties of the resulting boundary integral equations ahead from the discretization [5, 6, 1, 16, 31. Local approximations of the DtN map naturally define efficient analytical preconditioners. A strategy, developed in acoustic and electromagnetic scattering [7, 33], to obtain accurate local approximations of the DtN map is to consider only its principal symbol. Being able to derive such approximations in linear elasticity is a very challenging task due to the complexity of the three-dimensional elastic waves.

This paper is organized as follows. In Section 2, we introduce the problem setting and recall the Somigliana integral representation of the scattered field to justify the interest of defining approximate DtN maps. In Section 3, we derive exact DtN surface relations in the case of the elastic half-space using Fourier analysis tools which greatly simplify previous work lead by two of the authors [32. Section 4 is devoted to the construction of local approximations of the DtN map. The accuracy of the approximation is increased by a regularization process. Then the localization of the non-local operators is realized by using complex Padé approximants. In Section 5, we present a first possible application of this approximate DtN in the extreme OSRC context. Details on the finite element discretization and the numerical implementation are given together with various illustrations of the numerical efficiency. Finally, Section 6 gives some concluding remarks and directions for future work.

\section{Problem statement}

\subsection{Exterior Navier problem}

Let us consider a bounded domain $\Omega^{-}$in $\mathbb{R}^{3}$ representing an impenetrable body with a closed boundary $\Gamma:=\partial \Omega^{-}$of class $\mathscr{C}^{2}$ at least. Let $\Omega^{+}$denote the associated exterior domain $\mathbb{R}^{3} \backslash \overline{\Omega^{-}}$ and $\boldsymbol{n}$ the outer unit normal vector to the boundary $\Gamma$. The Lamé parameters $\mu$ and $\lambda$ and the density $\rho$ are positive constants. The propagation of time-harmonic waves in a three-dimensional isotropic and homogeneous elastic medium is modeled by the Navier equation

$$
\operatorname{div} \sigma(\boldsymbol{u})+\rho \omega^{2} \boldsymbol{u}=0
$$

where $\omega>0$ is the frequency. In the case of 3D isotropic elastodynamics, the stress and strain tensors are given by $\sigma(\boldsymbol{u})=\lambda(\operatorname{div} \boldsymbol{u}) \mathrm{I}_{3}+2 \mu \varepsilon(\boldsymbol{u})$ and $\varepsilon(\boldsymbol{u})=\frac{1}{2}\left([\boldsymbol{\nabla} \boldsymbol{u}]+[\boldsymbol{\nabla} \boldsymbol{u}]^{\top}\right)$ respectively, where $\mathrm{I}_{3}$ is the 3-by-3 identity matrix and $[\boldsymbol{\nabla} \boldsymbol{u}]$ is the matrix whose the $j$-th column is the gradient of the $j$-th component of $\boldsymbol{u}$. The field $\boldsymbol{u}$ can be decomposed into a longitudinal field $\boldsymbol{u}_{p}=\nabla \psi_{p}$ and a transverse field $\boldsymbol{u}_{s}=\boldsymbol{u}-\boldsymbol{u}_{p}=\operatorname{curl} \boldsymbol{\psi}_{s}$. The scalar and vector Lamé potentials $\psi_{p}$ and $\boldsymbol{\psi}_{s}$ satisfy 
respectively

$$
\left\{\begin{array} { c } 
{ \psi _ { p } = - \kappa _ { p } ^ { - 2 } \operatorname { d i v } \boldsymbol { u } } \\
{ \Delta \psi _ { p } + \kappa _ { p } ^ { 2 } \psi _ { p } = 0 }
\end{array} \quad \text { and } \quad \left\{\begin{array}{l}
\boldsymbol{\psi}_{s}=\kappa_{s}^{-2} \operatorname{curl} \boldsymbol{u} \\
\boldsymbol{\Delta} \boldsymbol{\psi}_{s}+\kappa_{s}^{2} \boldsymbol{\psi}_{s}=0
\end{array}\right.\right.
$$

where $\kappa_{p}^{2}=\rho \omega^{2}(\lambda+2 \mu)^{-1}$ and $\kappa_{s}^{2}=\rho \omega^{2} \mu^{-1}$ are the $\mathrm{P}$ and $\mathrm{S}$ wavenumbers. The wavelengths are defined by $\lambda_{\alpha}=2 \pi / \kappa_{\alpha}(\alpha=s, p)$. The scattering problem is formulated as follows : Given an incident wave field $\boldsymbol{u}^{\text {inc }}$ which is assumed to solve the Navier equation in the absence of any scatterer, find the displacement $\boldsymbol{u}$ solution to the Navier equation $(1)$ in $\Omega^{+}$which satisfies the Dirichlet boundary condition on $\Gamma$

$$
\boldsymbol{u}_{\mid \Gamma}+\boldsymbol{u}_{\mid \Gamma}^{\mathrm{inc}}=0
$$

In addition, the field $\boldsymbol{u}$ has to satisfy the Kupradze radiation conditions at infinity

$$
\lim _{r \rightarrow \infty} r\left(\frac{\partial \psi_{p}}{\partial r}-i \kappa_{p} \psi_{p}\right)=0, \quad \lim _{r \rightarrow \infty} r\left(\frac{\partial \boldsymbol{\psi}_{s}}{\partial r}-i \kappa_{s} \boldsymbol{\psi}_{s}\right)=0, \quad r=|\boldsymbol{x}|,
$$

uniformly in all directions.

\subsection{Traces and integral representation formula}

We denote by $H_{l o c}^{s}\left(\overline{\Omega^{+}}\right)$and $H^{s}(\Gamma)$ the standard (local in the case of the exterior domain) complex valued Hilbertian Sobolev spaces of order $s \in \mathbb{R}$ defined on $\overline{\Omega^{+}}$and $\Gamma$ respectively (with the convention $H^{0}=L^{2}$ ). Spaces of vector functions will be denoted by boldface letters, thus $\boldsymbol{H}^{s}=\left(H^{s}\right)^{3}$. We set $\Delta^{*} \boldsymbol{u}:=\operatorname{div} \sigma(\boldsymbol{u})=(\lambda+2 \mu) \boldsymbol{\nabla} \operatorname{div} \boldsymbol{u}-\mu$ curl curl $\boldsymbol{u}$ and introduce the energy space $\boldsymbol{H}_{+}^{1}\left(\Delta^{*}\right):=\left\{\boldsymbol{u} \in \boldsymbol{H}_{l o c}^{1}\left(\overline{\Omega^{+}}\right): \Delta^{*} \boldsymbol{u} \in \boldsymbol{L}_{l o c}^{2}\left(\overline{\Omega^{+}}\right)\right\}$. The Neumann trace, defined by $\boldsymbol{t}_{\mid \Gamma}:=\boldsymbol{T} \boldsymbol{u}$, is given by the traction operator

$$
\boldsymbol{T}=2 \mu \frac{\partial}{\partial \boldsymbol{n}}+\lambda \boldsymbol{n} \operatorname{div}+\mu \boldsymbol{n} \times \mathbf{c u r l} .
$$

We recall that we have $\boldsymbol{u}_{\mid \Gamma} \in \boldsymbol{H}^{\frac{1}{2}}(\Gamma)$ and $\boldsymbol{t}_{\mid \Gamma} \in \boldsymbol{H}^{-\frac{1}{2}}(\Gamma)$ for any $\boldsymbol{u} \in \boldsymbol{H}_{+}^{1}\left(\Delta^{*}\right)$. In the remaining of the paper we will use the tangential Günter derivative $\mathcal{M}$ defined by [45, Eq. (1.14) page 282]

$$
\mathcal{M}=\frac{\partial}{\partial \boldsymbol{n}}-\boldsymbol{n} \operatorname{div}+\boldsymbol{n} \times \mathbf{c u r l}
$$

We also use the surface differential operators: The tangential gradient $\nabla_{\Gamma}$, the surface divergence $\operatorname{div}_{\Gamma}$, the surface scalar curl $\operatorname{curl}_{\Gamma}$, the tangential vector curl $\operatorname{curl}_{\Gamma}$, the scalar Laplace-Beltrami operator $\Delta_{\Gamma}$ and the vector Laplace-Beltrami operator $\boldsymbol{\Delta}_{\Gamma}$. For their definitions we refer to [54, pages 68-75]. The tangential Günter derivative $\mathcal{M}$ is a surface derivative that can be rewritten

$$
\boldsymbol{M} \boldsymbol{u}_{\mid \Gamma}=\left(\left[\nabla_{\Gamma} \boldsymbol{u}_{\mid \Gamma}\right]-\left(\operatorname{div}_{\Gamma} \boldsymbol{u}_{\mid \Gamma}\right) \mathrm{I}_{3}\right) \boldsymbol{n}
$$

where $\left[\nabla_{\Gamma} \boldsymbol{v}\right]$ is the matrix whose the $j$-th column is the tangential gradient of the $j$-th component of $\boldsymbol{v}$. In the following, it is convenient to rewrite the traction operator as

$$
\boldsymbol{T}=2 \mu \mathcal{M}+(\lambda+2 \mu) \boldsymbol{n} \operatorname{div}-\mu \boldsymbol{n} \times \operatorname{curl} .
$$

As a result, the Neumann trace of the displacement field $\boldsymbol{u}$ is expressed in function of the Dirichlet trace of $\boldsymbol{u}$ and of the Lamé potentials $\psi_{p}$ and $\boldsymbol{\psi}_{s}$ as follows

$$
\boldsymbol{t}_{\mid \Gamma}=2 \mu \mathcal{M} \boldsymbol{u}_{\mid \Gamma}-\rho \omega^{2}\left(\boldsymbol{n}\left(\psi_{p}\right)_{\mid \Gamma}+\boldsymbol{n} \times\left(\boldsymbol{\psi}_{s}\right)_{\mid \Gamma}\right) .
$$

For a solution $\boldsymbol{u} \in \boldsymbol{H}_{+}^{1}\left(\Delta^{*}\right)$ to the Navier equation (1) in $\Omega^{+}$, that satisfies the Kupradze radiation conditions, the Somigliana integral representation of the field is given by

$$
\boldsymbol{u}(\boldsymbol{x})=\mathcal{D} \boldsymbol{u}_{\mid \Gamma}(\boldsymbol{x})-\mathcal{S} \boldsymbol{t}_{\mid \Gamma}(\boldsymbol{x}), \quad \boldsymbol{x} \in \Omega^{+},
$$


where the single- and double-layer potential operators are defined by

$$
\mathcal{S} \boldsymbol{\varphi}=\int_{\Gamma} \Phi(\cdot, \boldsymbol{y}) \boldsymbol{\varphi}(\boldsymbol{y}) d s(\boldsymbol{y}) \text { and } \mathcal{D} \boldsymbol{\psi}=\int_{\Gamma}\left[\boldsymbol{T}_{\boldsymbol{y}} \Phi(\cdot, \boldsymbol{y})\right]^{\top} \boldsymbol{\psi}(\boldsymbol{y}) d s(\boldsymbol{y}) .
$$

The fundamental solution of the Navier equation is a 3-by-3 matrix-valued function expressed by

$$
\Phi(\boldsymbol{x}, \boldsymbol{y})=\frac{1}{\rho \omega^{2}}\left(\operatorname{curl}_{\operatorname{curl}_{\boldsymbol{x}}}\left[\frac{e^{i \kappa_{s}|\boldsymbol{x}-\boldsymbol{y}|}}{4 \pi|\boldsymbol{x}-\boldsymbol{y}|} \mathrm{I}_{3}\right]-\boldsymbol{\nabla}_{\boldsymbol{x}} \operatorname{div}_{\boldsymbol{x}}\left[\frac{e^{i \kappa_{p}|\boldsymbol{x}-\boldsymbol{y}|}}{4 \pi|\boldsymbol{x}-\boldsymbol{y}|} \mathrm{I}_{3}\right]\right) .
$$

The tensor $\boldsymbol{T}_{\boldsymbol{y}} \Phi(\boldsymbol{x}, \boldsymbol{y})$ is obtained by applying the traction operator $\boldsymbol{T}_{\boldsymbol{y}}=\boldsymbol{T}\left(\boldsymbol{n}(\boldsymbol{y}), \partial_{\boldsymbol{y}}\right)$ to each column of $\Phi(\boldsymbol{x}, \boldsymbol{y})$. Due to the radiation conditions, the solution exhibits the following asymptotic behavior

$$
\boldsymbol{u}(\boldsymbol{x})=\frac{e^{i \kappa_{p}|\boldsymbol{x}|}}{|\boldsymbol{x}|} \boldsymbol{u}_{p}^{\infty}(\hat{\boldsymbol{x}})+\frac{e^{i \kappa_{s}|\boldsymbol{x}|}}{|\boldsymbol{x}|} \boldsymbol{u}_{s}^{\infty}(\hat{\boldsymbol{x}})+\mathcal{O}\left(\frac{1}{|\boldsymbol{x}|}\right), \quad|\boldsymbol{x}| \rightarrow+\infty,
$$

uniformly in all directions $\hat{\boldsymbol{x}}=\frac{\boldsymbol{x}}{|\boldsymbol{x}|}$. The fields $\boldsymbol{u}_{p}^{\infty}$ and $\boldsymbol{u}_{s}^{\infty}$, defined on the unit sphere $\mathbf{S}^{2}$ in $\mathbb{R}^{3}$, are the longitudinal and the transversal far-field patterns respectively. The far-field pattern is given by the integral representation formula

$$
\boldsymbol{u}^{\infty}(\hat{\boldsymbol{x}})=\mathscr{F}_{\mathrm{D}} \boldsymbol{u}_{\mid \Gamma}(\hat{\boldsymbol{x}})-\mathscr{F}_{\mathrm{S}} \boldsymbol{t}_{\mid \Gamma}(\hat{\boldsymbol{x}})
$$

where the far-field operators $\mathscr{F}_{\mathrm{S}}$ and $\mathscr{F}_{\mathrm{D}}$ are defined for any vector density $\varphi$ and $\hat{\boldsymbol{x}} \in \mathbf{S}^{2}[2]$ by

$$
\begin{gathered}
\mathscr{F}_{\mathrm{S}} \boldsymbol{\varphi}(\hat{\boldsymbol{x}})=\int_{\Gamma}\left(\frac{1}{\mu}\left[\mathrm{I}_{\mathbb{R}^{3}}-\hat{\boldsymbol{x}} \otimes \hat{\boldsymbol{x}}\right] \frac{e^{-i \kappa_{s} \hat{\boldsymbol{x}} \cdot \boldsymbol{y}}}{4 \pi}+\frac{1}{\lambda+2 \mu}[\hat{\boldsymbol{x}} \otimes \hat{\boldsymbol{x}}] \frac{e^{-i \kappa_{p} \hat{\boldsymbol{x}} \cdot \boldsymbol{y}}}{4 \pi}\right) \boldsymbol{\varphi}(\boldsymbol{y}) d s(\boldsymbol{y}) \text { and } \\
\mathscr{F}_{\mathrm{D}} \boldsymbol{\varphi}(\hat{\boldsymbol{x}})=\int_{\Gamma}\left(\frac{1}{\mu}\left[\boldsymbol{T}_{\boldsymbol{y}}\left[\mathrm{I}_{\mathbb{R}^{3}}-\hat{\boldsymbol{x}} \otimes \hat{\boldsymbol{x}}\right] \frac{e^{-i \kappa_{s} \hat{\boldsymbol{x}} \cdot \boldsymbol{y}}}{4 \pi}\right]^{\top}+\frac{1}{\lambda+2 \mu}\left[\boldsymbol{T}_{\boldsymbol{y}}[\hat{\boldsymbol{x}} \otimes \hat{\boldsymbol{x}}] \frac{e^{-i \kappa_{p} \hat{\boldsymbol{x}} \cdot y}}{4 \pi}\right]^{\top}\right) \boldsymbol{\varphi}(\boldsymbol{y}) d s(\boldsymbol{y}) .
\end{gathered}
$$

The new unknowns of the scattering problem are the surfacic data $\left(\boldsymbol{u}_{\mid \Gamma}, \boldsymbol{t}_{\mid \Gamma}\right)$. Several methods that are exact or approximate are proposed for computing the Neumann data $\boldsymbol{t}_{\mid \Gamma}$ knowing the Dirichlet one $\boldsymbol{u}_{\mid \Gamma}$, or conversely. Ideally, if we could explicit for a general shape the exact Dirichletto-Neumann (DtN) map which is defined by

$$
\begin{aligned}
\boldsymbol{\Lambda}^{\mathrm{ex}}: \boldsymbol{H}^{\frac{1}{2}}(\Gamma) & \rightarrow \boldsymbol{H}^{-\frac{1}{2}}(\Gamma), \\
\boldsymbol{u}_{\mid \Gamma} & \mapsto \boldsymbol{t}_{\mid \Gamma}
\end{aligned}
$$

the exterior problem (1)-(3)-(4) would be solved directly by the following representation

$$
\boldsymbol{u}(\boldsymbol{x})=-\left(\mathcal{D}-\mathcal{S} \boldsymbol{\Lambda}^{\mathrm{ex}}\right) \boldsymbol{u}_{\mid \Gamma}^{\mathrm{inc}}(\boldsymbol{x}), \quad \boldsymbol{x} \in \Omega^{+} .
$$

Unfortunately, this cannot be achieved for an arbitrary surface $\Gamma$. Instead, the idea is to derive a local approximate DtN map as accurate as possible. This approximation is usually reduced to a surface relation of the form

$$
\mathcal{P}_{1} \boldsymbol{u}_{\mid \Gamma}+\mathcal{P}_{2} \boldsymbol{t}_{\mid \Gamma}=0,
$$

where $\mathcal{P}_{1}$ and $\mathcal{P}_{2}$ are two invertible local boundary operators. The advantage of such operators is to lead after discretization to sparse matrices. Note that in $[15]$, the surfacic data $\boldsymbol{u}_{\mid \Gamma}$ and $\boldsymbol{t}_{\mid \Gamma}$ are not exact but approximate.

\section{Derivation of exact DtN surface relations for the elastic half-space}

Recent investigations in elastodynamics [32, based on the analysis of boundary integral operators and Calderón formulas, showed that the construction of the surface relation 15 is consid- 
erably simplified if a surface relation between the data $\boldsymbol{u}_{\mid \Gamma}$ and $\boldsymbol{t}_{\mid \Gamma}-2 \mu \mathcal{M} \boldsymbol{u}_{\mid \Gamma}$ of the form

$$
\mathcal{Q}_{1} \boldsymbol{u}_{\mid \Gamma}+\mathcal{Q}_{2}\left(\boldsymbol{t}_{\mid \Gamma}-2 \mu \mathcal{M} \boldsymbol{u}_{\mid \Gamma}\right)=0
$$

is considered. It follows that $\mathcal{P}_{1}=\mathcal{Q}_{1}-2 \mu \mathcal{Q}_{2} \mathcal{M}, \mathcal{P}_{2}=\mathcal{Q}_{2}$. For an elastic half-space, we can derive exact non-local boundary relations of type 15 and the exact DtN operator is given by

$$
\Lambda^{\mathrm{ex}}=-\mathcal{P}_{2}^{-1} \mathcal{P}_{1}=-\mathcal{Q}_{2}^{-1} \mathcal{Q}_{1}+2 \mu \mathcal{M} .
$$

In this section, we first construct the non-local boundary operators $\mathcal{Q}_{1}$ and $\mathcal{Q}_{2}$ using Fourier analysis. Then we present a physical interpretation of the proposed DtN surface relations.

\subsection{Derivation of some exact DtN operators using Fourier analysis}

Let us consider the case $\Gamma:=\left\{\boldsymbol{x} \in \mathbb{R}^{3} \mid x_{1}=0\right\}$. We denote by $\boldsymbol{n}:=(1,0,0)$ the outwardly directed unit normal vector at $\Gamma$ to $\Omega^{-}:=\left\{\boldsymbol{x} \in \mathbb{R}^{3} \mid x_{1}<0\right\}$. Assuming that $x_{1}$ is the radial direction to $\Omega^{-}$, then the tangential direction is $\boldsymbol{x}_{\|}:=\left(x_{2}, x_{3}\right)$. We define the partial Fourier transform $\hat{\boldsymbol{f}}$ of a function $\boldsymbol{f}:=\left(f_{1}, f_{2}, f_{3}\right): \mathbb{R}^{3} \rightarrow \mathbb{R}^{3}$ by

$$
\hat{f}_{j}\left(x_{1}, \xi\right):=\int_{\mathbb{R}^{2}} f_{j}\left(x_{1}, \boldsymbol{x}_{\|}\right) e^{-i \boldsymbol{x}_{\|} \cdot \boldsymbol{\xi}} d \boldsymbol{x}_{\|}, \quad \text { for } j=2,3,
$$

and the inverse Fourier transform by

$$
f_{j}(\boldsymbol{x}):=\frac{1}{2 \pi} \int_{\mathbb{R}^{2}} \hat{f}_{j}\left(x_{1}, \boldsymbol{\xi}\right) e^{i \boldsymbol{x}_{\|} \cdot \boldsymbol{\xi}} d \boldsymbol{\xi}, \quad \text { for } j=2,3 .
$$

The dual variable of $\boldsymbol{x}_{\|}$is $\boldsymbol{\xi}:=\left(\xi_{2}, \xi_{3}\right)$ and we set $\|\boldsymbol{\xi}\|:=\sqrt{\boldsymbol{\xi} \cdot \boldsymbol{\xi}}$.

Proposition 3.1. For $\Gamma=\left\{\boldsymbol{x} \in \mathbb{R}^{3} \mid x_{1}=0\right\}$, we have the following equivalent exact surface relations :

(i) $\boldsymbol{u}_{\mid \Gamma}=\mathbf{V}\left(\boldsymbol{t}_{\mid \Gamma}-2 \mu \mathcal{M} \boldsymbol{u}_{\mid \Gamma}\right)$, with

$$
\mathbf{V}=\left(-\frac{1}{\rho \omega^{2}} \mathcal{M}+\frac{1}{i \rho \omega^{2}}\left(\boldsymbol{n}\left(\Delta_{\Gamma}+\kappa_{p}^{2} \mathrm{I}\right)^{\frac{1}{2}} \boldsymbol{n} \cdot \mathrm{I}_{\boldsymbol{n}}+\left(\boldsymbol{\Delta}_{\Gamma}+\kappa_{s}^{2} \mathrm{I}_{\boldsymbol{t}}\right)^{-\frac{1}{2}}\left(\kappa_{s}^{2} \mathrm{I}_{\boldsymbol{t}}+\boldsymbol{\nabla}_{\Gamma} \operatorname{div}_{\Gamma} \mathrm{I}_{\boldsymbol{t}}\right)\right)\right)
$$

(ii) $\boldsymbol{\Lambda}_{\mathbf{1}} \boldsymbol{u}_{\mid \Gamma}=\left(\mathrm{I}+\boldsymbol{\Lambda}_{\mathbf{2}}\right)\left(\boldsymbol{t}_{\mid \Gamma}-2 \mu \mathcal{M} \boldsymbol{u}_{\mid \Gamma}\right)$, with

$$
\begin{aligned}
& \boldsymbol{\Lambda}_{\mathbf{1}}=i \rho \omega^{2}\left[\boldsymbol{n}\left(\Delta_{\Gamma}+\kappa_{p}^{2} \mathrm{I}\right)^{-\frac{1}{2}} \boldsymbol{n} \cdot \mathrm{I}_{\boldsymbol{n}}+\left(\boldsymbol{\Delta}_{\Gamma}+\kappa_{s}^{2} \mathrm{I}_{\boldsymbol{t}}\right)^{-\frac{1}{2}}\left(\mathrm{I}_{\boldsymbol{t}}-\frac{1}{\kappa_{s}^{2}} \operatorname{curl}_{\Gamma} \operatorname{curl}_{\Gamma}\right)\right] \\
& \text { and } \\
& \boldsymbol{\Lambda}_{\mathbf{2}}=-i\left(\boldsymbol{\nabla}_{\Gamma}\left(\Delta_{\Gamma}+\kappa_{s}^{2} \mathrm{I}\right)^{-\frac{1}{2}} \boldsymbol{n} \cdot \mathrm{I}_{\boldsymbol{n}}-\boldsymbol{n}\left(\Delta_{\Gamma}+\kappa_{p}^{2} \mathrm{I}\right)^{-\frac{1}{2}} \operatorname{div}_{\Gamma} \mathrm{I}_{\boldsymbol{t}}\right),
\end{aligned}
$$

where we have defined $\mathrm{I}_{\boldsymbol{n}}=\boldsymbol{n} \otimes \boldsymbol{n}, \mathrm{I}_{\boldsymbol{t}}=\mathrm{I}-\mathrm{I}_{\boldsymbol{n}}$.

Proof. • In a first step, we rewrite the elastodynamic system (1) as a first-order hyperbolic system where the unknown is the Fourier transform of

$$
\boldsymbol{U}=\left(\boldsymbol{u},(\lambda+2 \mu) \operatorname{div} \boldsymbol{u}, \mu(\operatorname{curl} \boldsymbol{u})_{3},-\mu(\operatorname{curl} \boldsymbol{u})_{2}\right)^{\top},
$$

setting $\boldsymbol{u}=\left(u_{1}, u_{2}, u_{3}\right)$. The Dirichlet trace of $\boldsymbol{U}$ is given by $\boldsymbol{U}_{\mid \Gamma}=\left(\boldsymbol{u}_{\mid \Gamma}, \boldsymbol{t}_{\mid \Gamma}-2 \mu \mathcal{M} \boldsymbol{u}_{\mid \Gamma}\right)^{\top}$ with $\boldsymbol{t}_{\mid \Gamma}-2 \mu \boldsymbol{M} \boldsymbol{u}_{\mid \Gamma}=-\rho \omega^{2}\left(\boldsymbol{n}\left(\psi_{p}\right)_{\mid \Gamma}+\boldsymbol{n} \times\left(\boldsymbol{\psi}_{s}\right)_{\mid \Gamma}\right)$. 
If we develop system (1), we obtain

$$
\left\{\begin{array}{c}
\partial_{1}(\lambda+2 \mu) \operatorname{div} \boldsymbol{u}-\partial_{2}\left(\mu(\operatorname{curl} \boldsymbol{u})_{3}\right)-\partial_{3}\left(-\mu(\operatorname{curl} \boldsymbol{u})_{2}\right)+\rho \omega^{2} u_{1}=0 \\
\partial_{2}(\lambda+2 \mu) \operatorname{div} \boldsymbol{u}-\partial_{3}\left(\mu(\operatorname{curl} \boldsymbol{u})_{1}\right)+\partial_{1}\left(\mu(\operatorname{curl} \boldsymbol{u})_{3}\right)+\rho \omega^{2} u_{2}=0 \\
\partial_{3}(\lambda+2 \mu) \operatorname{div} \boldsymbol{u}+\partial_{1}\left(-\mu(\operatorname{curl} \boldsymbol{u})_{2}\right)+\partial_{2}\left(\mu(\operatorname{curl} \boldsymbol{u})_{1}\right)+\rho \omega^{2} u_{3}=0
\end{array}\right.
$$

Using the equality $(\operatorname{curl} \boldsymbol{u})_{1}=\partial_{2} u_{3}-\partial_{3} u_{2}$ and rearranging $[19$, we obtain

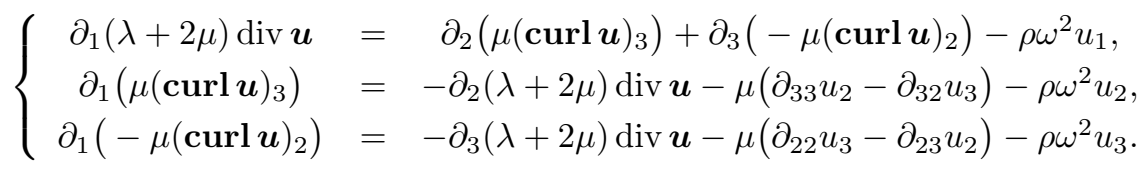

Following [19), we compute the first derivative of $\boldsymbol{u}$ with respect to $x_{1}$

$$
\left\{\begin{array}{l}
\partial_{1} u_{1}=-\frac{1}{\rho \omega^{2}}\left(\partial_{11}^{2}(\lambda+2 \mu) \operatorname{div} \boldsymbol{u}-\partial_{21}^{2}\left(\mu(\operatorname{curl} \boldsymbol{u})_{3}\right)-\partial_{31}^{2}\left(-\mu(\operatorname{curl} \boldsymbol{u})_{2}\right)\right) \\
\partial_{1} u_{2}=-\frac{1}{\rho \omega^{2}}\left(\partial_{21}^{2}(\lambda+2 \mu) \operatorname{div} \boldsymbol{u}-\partial_{13}^{2}\left(\mu(\operatorname{curl} \boldsymbol{u})_{1}\right)+\partial_{11}^{2}\left(\mu(\operatorname{curl} \boldsymbol{u})_{3}\right)\right) \\
\partial_{1} u_{3}=-\frac{1}{\rho \omega^{2}}\left(\partial_{31}^{2}(\lambda+2 \mu) \operatorname{div} \boldsymbol{u}+\partial_{11}^{2}\left(-\mu(\operatorname{curl} \boldsymbol{u})_{2}\right)+\partial_{12}^{2}\left(\mu(\operatorname{curl} \boldsymbol{u})_{1}\right)\right)
\end{array}\right.
$$

To eliminate the second order derivative with respect to $x_{1}$, we use equalities (2) to obtain

$$
\left\{\begin{aligned}
\partial_{1} u_{1}= & \frac{1}{\rho \omega^{2}}\left(\kappa_{p}^{2}+\partial_{22}^{2}+\partial_{33}^{2}\right)(\lambda+2 \mu) \operatorname{div} \boldsymbol{u} \\
& -\frac{1}{\rho \omega^{2}}\left(-\partial_{21}^{2}\left(\mu(\operatorname{curl} \boldsymbol{u})_{3}\right)-\partial_{31}^{2}\left(-\mu(\operatorname{curl} \boldsymbol{u})_{2}\right)\right), \\
\partial_{1} u_{2}= & -\frac{1}{\rho \omega^{2}}\left(-\partial_{13}^{2}\left(\mu(\operatorname{curl} \boldsymbol{u})_{1}\right)-\left(\kappa_{s}^{2}+\partial_{22}^{2}+\partial_{33}^{2}\right)\left(\mu(\operatorname{curl} \boldsymbol{u})_{3}\right)\right) \\
& -\frac{1}{\rho \omega^{2}} \partial_{21}^{2}(\lambda+2 \mu) \operatorname{div} \boldsymbol{u}, \\
\partial_{1} u_{3}= & -\frac{1}{\rho \omega^{2}}\left(-\left(\kappa_{s}^{2}+\partial_{22}^{2}+\partial_{33}^{2}\right)\left(-\mu(\operatorname{curl} \boldsymbol{u})_{2}\right)+\partial_{12}^{2}\left(\mu(\operatorname{curl} \boldsymbol{u})_{1}\right)\right) \\
& -\frac{1}{\rho \omega^{2}} \partial_{31}^{2}(\lambda+2 \mu) \operatorname{div} \boldsymbol{u} .
\end{aligned}\right.
$$

Noting that

$$
\partial_{13}^{2}(\operatorname{curl} \boldsymbol{u})_{1}=-\partial_{23}^{2}(\operatorname{curl} \boldsymbol{u})_{2}-\partial_{33}^{2}(\operatorname{curl} \boldsymbol{u})_{3}
$$

and

$$
\partial_{12}^{2}(\operatorname{curl} \boldsymbol{u})_{1}=-\partial_{22}^{2}(\operatorname{curl} \boldsymbol{u})_{2}-\partial_{23}^{2}(\operatorname{curl} \boldsymbol{u})_{3}
$$

we get

$$
\left\{\begin{aligned}
\partial_{1} u_{1}= & \frac{1}{\rho \omega^{2}}\left(\kappa_{p}^{2}+\partial_{22}^{2}+\partial_{33}^{2}\right)(\lambda+2 \mu) \operatorname{div} \boldsymbol{u} \\
& -\frac{1}{\rho \omega^{2}}\left(-\partial_{21}^{2}\left(\mu(\operatorname{curl} \boldsymbol{u})_{3}\right)-\partial_{31}^{2}\left(-\mu(\operatorname{curl} \boldsymbol{u})_{2}\right)\right) \\
\partial_{1} u_{2}= & -\frac{1}{\rho \omega^{2}}\left(-\partial_{23}^{2}\left(-\mu(\operatorname{curl} \boldsymbol{u})_{2}\right)-\left(\kappa_{s}^{2}+\partial_{22}^{2}\right)\left(\mu(\operatorname{curl} \boldsymbol{u})_{3}\right)\right) \\
& -\frac{1}{\rho \omega^{2}} \partial_{21}^{2}(\lambda+2 \mu) \operatorname{div} \boldsymbol{u} \\
\partial_{1} u_{3}= & -\frac{1}{\rho \omega^{2}}\left(-\left(\kappa_{s}^{2}+\partial_{33}^{2}\right)\left(-\mu(\operatorname{curl} \boldsymbol{u})_{2}\right)-\partial_{23}^{2}\left(\mu(\operatorname{curl} \boldsymbol{u})_{3}\right)\right) \\
& -\frac{1}{\rho \omega^{2}} \partial_{31}^{2}(\lambda+2 \mu) \operatorname{div} \boldsymbol{u} .
\end{aligned}\right.
$$

Applying the partial Fourier transform along $\boldsymbol{x}_{\|}$to systems (20) and (21), we have the equation 
$\mathbf{A} \partial_{x_{1}} \widehat{U}=\mathbf{B} \widehat{U}$ where

$$
\mathbf{A}=\left(\begin{array}{cc}
\mathbf{I}_{\mathbf{3}} & \frac{1}{\rho \omega^{2}} \mathbf{M} \\
\mathbf{0}_{3} & \mathbf{I}_{\mathbf{3}}
\end{array}\right) \quad \text { with } \quad \mathbf{M}=\left(\begin{array}{ccc}
0 & -i \xi_{2} & -i \xi_{3} \\
i \xi_{2} & 0 & 0 \\
i \xi_{3} & 0 & 0
\end{array}\right)
$$

and

$$
\mathbf{B}=\left(\begin{array}{cccccc}
0 & 0 & 0 & \frac{\kappa_{p}^{2}-\|\xi\|^{2}}{\rho \omega^{2}} & 0 & 0 \\
0 & 0 & 0 & 0 & \frac{\kappa_{s}^{2}-\xi_{2}^{2}}{\rho \omega^{2}} & -\frac{\xi_{2} \xi_{3}}{\rho \omega^{2}} \\
0 & 0 & 0 & 0 & -\frac{\xi_{2} \xi_{3}}{\rho \omega^{2}} & \frac{\kappa_{s}^{2}-\xi_{3}^{2}}{\rho \omega^{2}} \\
-\rho \omega^{2} & 0 & 0 & 0 & i \xi_{2} & i \xi_{3} \\
0 & -\left(\rho \omega^{2}-\mu \xi_{3}^{2}\right) & -\mu \xi_{2} \xi_{3} & -i \xi_{2} & 0 & 0 \\
0 & -\mu \xi_{2} \xi_{3} & -\left(\rho \omega^{2}-\mu \xi_{2}^{2}\right) & -i \xi_{3} & 0 & 0
\end{array}\right)
$$

- To solve the hyperbolic equation, we introduce the change of variable $\boldsymbol{V}=\mathbf{A} \widehat{\boldsymbol{U}}$ and write $\partial_{x_{1}} \boldsymbol{V}=\mathbf{B A}^{-1} \boldsymbol{V}$. Setting $s_{p}=\sqrt{\kappa_{p}^{2}-\|\boldsymbol{\xi}\|^{2}}$ and $s_{s}=\sqrt{\kappa_{s}^{2}-\|\boldsymbol{\xi}\|^{2}}$, the eigenvalues of the matrix $\mathbf{B A}^{-1}$ are $\pm i s_{p}$ with multiplicity 1 and $\pm i s_{s}$ with multiplicity 2 . The matrix $\mathbf{B A}^{-1}$ is diagonalized as $\mathbf{B} \mathbf{A}^{-1}=\mathbf{P D P}^{-1}$ with $\mathbf{D}=\operatorname{diag}\left(-i s_{p},-i s_{s},-i s_{s}, i s_{p}, i s_{s}, i s_{s}\right)$. The associated matrix $\mathbf{P}$ is

$$
\mathbf{P}=\left(\begin{array}{cc}
\mathbf{I}_{3} & \mathbf{I}_{3} \\
-\mathbf{C} & \mathbf{C}
\end{array}\right) \text { with } \mathbf{C}=i \rho \omega^{2}\left(\begin{array}{ccc}
s_{p}^{-1} & 0 & 0 \\
0 & \frac{\left(\kappa_{s}^{2}-\xi_{3}^{2}\right)}{\kappa_{s}^{2} s_{s}} & \frac{\xi_{2} \xi_{3}}{\kappa_{s}^{2} s_{s}} \\
0 & \frac{\xi_{2} \xi_{3}}{\kappa_{s}^{2} s_{s}} & \frac{\left(\kappa_{s}^{2}-\xi_{3}^{2}\right)}{\kappa_{s}^{2} s_{s}}
\end{array}\right)
$$

The first-order hyperbolic system is accordingly rewritten as $\partial_{x_{1}} \boldsymbol{W}=\mathbf{D} \boldsymbol{W}$, for $x_{1} \geq 0$, where $\boldsymbol{W}=\mathbf{P}^{-1} \boldsymbol{V}=\mathbf{P}^{-1} \mathbf{A} \widehat{\boldsymbol{U}}$. The solution of this equation is $\boldsymbol{W}\left(x_{1}\right)=e^{x_{1} D} \boldsymbol{W}(0)$. Since we are characterizing the part of the wave field that is square integrable in the right half-space, then we must impose that the first three components of $\boldsymbol{W}(0)$ vanish. Coming back to $\boldsymbol{U}$, we obtain the equation

$$
\widehat{\boldsymbol{u} \mid \Gamma}=\left(-\frac{1}{\rho \omega^{2}} \mathbf{M}+\mathbf{C}^{-1}\right)\left(\widehat{\boldsymbol{t}_{\mid \Gamma}}-2 \mu \mathbf{M} \widehat{\boldsymbol{u} \mid \Gamma}\right) .
$$

Applying the inverse Fourier transform we obtain surface relation $(i)$.

Another choice for the eigenfunctions (by imposing the unitary coordinate on the last three coordinates) would yield the following equation

$$
\mathbf{C} \widehat{\boldsymbol{u}_{\mid \Gamma}}=\left(-\frac{1}{\rho \omega^{2}} \mathbf{C M}+\mathbf{I}_{3}\right)\left(\widehat{\boldsymbol{t}_{\mid \Gamma}}-2 \mu \mathbf{M} \widehat{\boldsymbol{u} \mid \Gamma}\right) .
$$

Applying the inverse Fourier transform, we get surface relation (ii).

We have obtained two expressions (that are equivalent) for the DtN map $\boldsymbol{\Lambda}^{\mathrm{ex}}$. The boundary condition (ii) corresponds to the approximate DtN operator obtained in 32] for a general smooth surface through the computations of the principal parts of the elementary boundary integral operators. The alternative proof proposed here using Fourier analysis is more straightforward and falls into the techniques used for OSRC method [3, 7, 33].

\subsection{Physical interpretation}

The DtN operators proposed in Proposition 3.1 may seem complicated compared to the ones constructed in acoustics or electromagnetism but it is due to the definition of elastic waves in an isotropic and homogeneous medium. Indeed, using relations (2) and (6) between the displacement 
field $\boldsymbol{u}$ and the Lamé potentials $\psi_{p}$ and $\boldsymbol{\psi}_{s}$, we retrieve the surface relations of Proposition 3.1 as follows:

Proposition 3.2. Assume $\Gamma=\left\{\boldsymbol{x} \in \mathbb{R}^{3} \mid x_{1}=0\right\}$. Let $\boldsymbol{\Lambda}_{A}^{\mathrm{ex}}$ be the exact acoustic DtN operator for the P-wavenumber $\kappa_{p}$ and $\boldsymbol{\Lambda}_{E M}^{\mathrm{ex}}$ be the exact Magnetic-to-Electric (MtE) operator for the $S$ wavenumber $\kappa_{s}$, which are defined by (see [7, Equation (11)] and [33, Proposition 1] with $Z_{0}=1$ )

$$
\boldsymbol{\Lambda}_{A}^{\mathrm{ex}}=i\left(\Delta_{\Gamma}+\kappa_{p}^{2} \mathrm{I}\right)^{\frac{1}{2}} \quad \text { and } \quad \boldsymbol{\Lambda}_{E M}^{\mathrm{ex}}=\left(\mathrm{I}_{\boldsymbol{t}}+\frac{1}{\kappa_{s}^{2}} \Delta_{\Gamma}\right)^{-\frac{1}{2}}\left(\mathrm{I}_{\boldsymbol{t}}-\frac{1}{\kappa_{s}^{2}} \operatorname{curl}_{\Gamma} \operatorname{curl}_{\Gamma}\right)
$$

such that

$$
\begin{gathered}
\boldsymbol{\Lambda}_{A}^{\mathrm{ex}}\left(\psi_{p}\right)_{\mid \Gamma}=\frac{\partial}{\partial \boldsymbol{n}} \psi_{p} \quad \text { and } \quad \boldsymbol{\Lambda}_{E M}^{\mathrm{ex}}\left(\boldsymbol{n} \times\left(\left(\boldsymbol{\psi}_{s}\right)_{\mid \Gamma} \times \boldsymbol{n}\right)\right)=-\frac{1}{i \kappa_{s}} \boldsymbol{n} \times\left(\operatorname{curl} \boldsymbol{\psi}_{s}\right)_{\mid \Gamma}, \\
\left(\psi_{p}\right)_{\mid \Gamma}=\left(\boldsymbol{\Lambda}_{A}^{\mathrm{ex}}\right)^{-1} \frac{\partial}{\partial \boldsymbol{n}} \psi_{p} \quad \text { and } \quad \boldsymbol{\Lambda}_{E M}^{\mathrm{ex}}\left(\boldsymbol{n} \times\left(\left(\operatorname{curl} \boldsymbol{\psi}_{s}\right)_{\mid \Gamma} \times \boldsymbol{n}\right)\right)=i \kappa_{s} \boldsymbol{n} \times\left(\boldsymbol{\psi}_{s}\right)_{\mid \Gamma} .
\end{gathered}
$$

Then we have

$$
\begin{aligned}
& \text { (i) } \boldsymbol{u}_{\mid \Gamma}=-\frac{1}{\rho \omega^{2}}\left[\mathcal{M}+\boldsymbol{n} \boldsymbol{\Lambda}_{A}^{\mathrm{ex}}\left(\boldsymbol{n} \cdot \mathrm{I}_{\boldsymbol{n}}\right)+i \kappa_{s} \boldsymbol{n} \times \boldsymbol{\Lambda}_{E M}^{\mathrm{ex}}\left(\mathrm{I}_{\boldsymbol{t}} \times \boldsymbol{n}\right)\right]\left(\boldsymbol{t}_{\mid \Gamma}-2 \mu \mathcal{M} \boldsymbol{u}_{\mid \Gamma}\right) \text {. } \\
& \text { (ii) }\left[\boldsymbol{n}\left(\boldsymbol{\Lambda}_{A}^{\mathrm{ex}}\right)^{-1}\left(\boldsymbol{n} \cdot \mathrm{I}_{\boldsymbol{n}}\right)+\frac{1}{i \kappa_{s}} \boldsymbol{\Lambda}_{E M}^{\mathrm{ex}} \mathrm{I}_{\boldsymbol{t}}\right] \boldsymbol{u}_{\mid \Gamma} \\
& =-\frac{1}{\rho \omega^{2}}\left[\mathrm{I}-\boldsymbol{n}\left(\boldsymbol{\Lambda}_{A}^{\mathrm{ex}}\right)^{-1} \operatorname{div}_{\Gamma} \mathrm{I}_{\boldsymbol{t}}+\frac{1}{i \kappa_{s}} \boldsymbol{\Lambda}_{E M}^{\mathrm{ex}} \nabla_{\Gamma}\left(\boldsymbol{n} \cdot \mathrm{I}_{\boldsymbol{n}}\right)\right]\left(\boldsymbol{t}_{\mid \Gamma}-2 \mu \boldsymbol{M} \boldsymbol{u}_{\mid \Gamma}\right) .
\end{aligned}
$$

Proof. First, we recall that for any scalar function $v \in \mathscr{C}^{1}\left(\overline{\Omega^{+}}\right)$and any vector function $\boldsymbol{w} \in$ $\left(\mathscr{C}^{1}\left(\overline{\Omega^{+}}\right)\right)^{3}$, the following identities hold on the boundary $\Gamma$

$$
\nabla_{\Gamma} v_{\mid \Gamma}=\boldsymbol{n} \times\left((\nabla v)_{\mid \Gamma} \times \boldsymbol{n}\right) \quad \text { and } \quad \frac{\partial}{\partial \boldsymbol{n}} v=\boldsymbol{n} \cdot(\nabla v)_{\mid \Gamma},
$$

and

$$
\boldsymbol{n} \cdot(\operatorname{curl} \boldsymbol{w})_{\mid \Gamma}=\operatorname{curl}_{\Gamma} \boldsymbol{w}=-\operatorname{div}_{\Gamma}\left(\boldsymbol{n} \times \boldsymbol{w}_{\mid \Gamma}\right) .
$$

The decomposition $\boldsymbol{u}=\boldsymbol{\nabla} \psi_{p}+\operatorname{curl} \psi_{s}$ leads to

$$
\left\{\begin{array}{cl}
\boldsymbol{n} \cdot \boldsymbol{u}_{\mid \Gamma} & =\boldsymbol{n} \cdot\left(\boldsymbol{\nabla} \psi_{p}\right)_{\mid \Gamma}+\boldsymbol{n} \cdot\left(\operatorname{curl} \boldsymbol{\psi}_{s}\right)_{\mid \Gamma} \\
& =\frac{\partial}{\partial \boldsymbol{n}} \psi_{p}-\operatorname{div}_{\Gamma}\left(\boldsymbol{n} \times\left(\boldsymbol{\psi}_{s}\right)_{\mid \Gamma}\right) \\
\boldsymbol{n} \times\left(\boldsymbol{u}_{\mid \Gamma} \times \boldsymbol{n}\right) & =\boldsymbol{\nabla}_{\Gamma}\left(\psi_{p}\right)_{\mid \Gamma}+\boldsymbol{n} \times\left(\left(\operatorname{curl} \boldsymbol{\psi}_{s}\right)_{\mid \Gamma} \times \boldsymbol{n}\right),
\end{array}\right.
$$

and from (6) we deduce

$$
\left\{\begin{array}{ccc}
\boldsymbol{n} \cdot\left(\boldsymbol{t}_{\mid \Gamma}-2 \mu \mathcal{M} \boldsymbol{u}_{\mid \Gamma}\right) & = & -\rho \omega^{2}\left(\psi_{p}\right)_{\mid \Gamma} \\
\boldsymbol{n} \times\left(\left(\boldsymbol{t}_{\mid \Gamma}-2 \mu \mathcal{M} \boldsymbol{u}_{\mid \Gamma}\right) \times \boldsymbol{n}\right) & = & -\rho \omega^{2} \boldsymbol{n} \times\left(\boldsymbol{\psi}_{s}\right)_{\mid \Gamma} .
\end{array}\right.
$$

We obtain the following rewritting of the boundary data $\boldsymbol{u}_{\mid \Gamma}$ and $\boldsymbol{t}_{\mid \Gamma}-2 \mu \mathcal{M} \boldsymbol{u}_{\mid \Gamma}$

$$
-\frac{1}{\rho \omega^{2}}\left(\boldsymbol{t}_{\mid \Gamma}-2 \mu \mathcal{M} \boldsymbol{u}_{\mid \Gamma}\right)=\boldsymbol{n}\left(\psi_{p}\right)_{\mid \Gamma}+\boldsymbol{n} \times\left(\boldsymbol{\psi}_{s}\right)_{\mid \Gamma}
$$

and

$$
\boldsymbol{u}_{\mid \Gamma}=\boldsymbol{n} \frac{\partial}{\partial \boldsymbol{n}} \psi_{p}-\boldsymbol{n} \operatorname{div}_{\Gamma}\left(\boldsymbol{n} \times\left(\boldsymbol{\psi}_{s}\right)_{\mid \Gamma}\right)+\boldsymbol{\nabla}_{\Gamma}\left(\psi_{p}\right)_{\mid \Gamma}+\boldsymbol{n} \times\left(\left(\operatorname{curl} \boldsymbol{\psi}_{s}\right)_{\mid \Gamma} \times \boldsymbol{n}\right)
$$


Using that on the boundary $\Gamma$ of a half-space

$$
\mathcal{M}=\nabla_{\Gamma}\left(\boldsymbol{n} \cdot \mathrm{I}_{\boldsymbol{n}}\right)-\operatorname{div}_{\Gamma} \mathrm{I}_{\boldsymbol{t}}
$$

and substituting 22 in the expression of $\boldsymbol{u}_{\mid \Gamma}$, the relation $(i)$ comes. Applying the operator $\left[\boldsymbol{n}\left(\boldsymbol{\Lambda}_{A}^{\mathrm{ex}}\right)^{-1}\left(\boldsymbol{n} \cdot \mathrm{I}_{\boldsymbol{n}}\right)+\frac{1}{i \kappa_{s}} \boldsymbol{\Lambda}_{E M}^{\mathrm{ex}} \mathrm{I}_{\boldsymbol{t}}\right]$ to $\boldsymbol{u}_{\mid \Gamma}$ and using $[23)$, we obtain relation (ii).

\section{Local approximate DtN surface operators for a general smooth surface $\Gamma$}

The boundary relations given in Proposition 3.1 are non local and, are valid only in the case of an elastic half-space. There exists two ways to construct such surface operators in the case of a general smooth surface: a formal one using a tangent plane approximation and a rigourous one applying the technique of microlocal diagonalization for hyperbolic systems 34. These two approaches allow to obtain the surface approximations $(i)$ and $(i i)$ in Proposition (3.1) onto a general smooth surface $\Gamma$. In the sequel, we choose to consider the approximation (ii) that is more judicious numerically speaking. Indeed, both surface relations require to invert some surface differential operators. The GMRES solver is chosen to compute the solution of the associated linear systems (see Section 5). The operator $\mathbf{V}$ in $(i)$ is of order +1 and the operator $\left(\mathrm{I}+\boldsymbol{\Lambda}_{\mathbf{2}}\right)$ in (ii) is of order 0 . The latter is thus more appropriate.

In this section, we first explain how to improve the accuracy of the approximate DtN operators using a regularization procedure and then, how to localize these operators with a complex Padé rational approximation of the square-root.

\subsection{Regularization of the approximate DtN map}

Let us consider the time-harmonic scattering problem of a plane wave by a rigid elastic sphere of radius $R>0$. In this case, the Neumann trace $\boldsymbol{t}_{\mid \Gamma}$ of the displacement $\boldsymbol{u}$ solution to the exterior problem (1)-(3)-(4) is computed analytically in terms of vector spherical harmonics. We also compute an approximate Neumann trace by applying the surface relation (ii) in Proposition 3.1 as an OSRC equation directly on the surface of a rigid elastic sphere, namely

$$
\tilde{\boldsymbol{t}}_{\mid \Gamma}=\left(\left(\mathrm{I}+\boldsymbol{\Lambda}_{\mathbf{2}}\right)^{-1} \boldsymbol{\Lambda}_{\mathbf{1}}+2 \mu \mathcal{M}\right) \boldsymbol{u}_{\mid \Gamma}
$$

The comparison of these two surfacic data gives a first indication on the accuracy of the proposed approximate DtN operator in $(i i)$. We consider incident plane waves of the form

$$
\boldsymbol{u}^{\text {inc }}(\boldsymbol{x})=\frac{1}{\mu} e^{i \kappa_{s} \boldsymbol{x} \cdot \boldsymbol{d}}(\boldsymbol{d} \times \boldsymbol{p}) \times \boldsymbol{d}+\frac{1}{\lambda+2 \mu} e^{i \kappa_{p} \boldsymbol{x} \cdot \boldsymbol{d}}(\boldsymbol{d} \cdot \boldsymbol{p}) \boldsymbol{d}, \quad \text { where } \boldsymbol{d} \in \mathbf{S}^{2} \text { and } \boldsymbol{p} \in \mathbb{R}^{3} .
$$

When $\boldsymbol{p}= \pm \boldsymbol{d}$, the incident plane wave oscillates along the direction of propagation (pressure wave or P-wave). When the polarization $\boldsymbol{p}$ is orthogonal to the propagation vector $\boldsymbol{d}$, the incident plane wave oscillates in a direction orthogonal to the direction of propagation (shear wave or S-wave). Such incidence plane waves admit the following series expansion

$$
\boldsymbol{u}^{\mathrm{inc}}(\boldsymbol{x})=\sum_{m=1}^{\infty} \sum_{j=-m}^{m}\left(\alpha_{m}^{(1)} M_{m, j}^{(1)}\left(\kappa_{s}, \boldsymbol{x}\right)+\beta_{m}^{(1)} N_{m, j}^{(1)}\left(\kappa_{s}, \boldsymbol{x}\right)\right)+\sum_{m=0}^{\infty} \sum_{j=-m}^{m} \gamma_{m}^{(1)} \nabla u_{m, j}^{(1)}\left(\kappa_{p}, \boldsymbol{x}\right) .
$$

The scalar function $u_{m, j}^{(k)}\left(\kappa_{p}, \boldsymbol{x}\right)$, the vector functions $M_{m, j}^{(k)}\left(\kappa_{s}, \boldsymbol{x}\right)$ and $N_{m, j}^{(k)}\left(\kappa_{s}, \boldsymbol{x}\right)$ of order $m$ for $j=-m, \ldots, m$, with $m, j \in \mathbb{N}$, and the spectral coefficients are given in the Appendix. The spectral decomposition of both the Neumann trace $\boldsymbol{t}_{\mid \Gamma}$ and its approximation $\tilde{\boldsymbol{t}}_{\mid \Gamma}$ are also proposed in the Appendix (cf. formulas A.1 and A.2 respectively). To analyze the accuracy of the approximate DtN operator in the surface relation $(i i)$, we compare these two analytical solutions. We report on Figure 1 the modulus of the coefficients $\alpha_{m}^{\text {ex }}$ and $\alpha_{m}^{\text {app }}$ (left), $\beta_{m}^{\text {ex }}$ and $\beta_{m}^{\text {app }}$ (center), $\gamma_{m}^{\text {ex }}$ and $\gamma_{m}^{\text {app }}$ (right) as a function of the modes $m$. In all the simulations, the mechanical 
parameters are chosen such that the wavenumbers satisfy $\kappa_{s}=2 \kappa_{p}$. We consider an incident plane $S$-wave with $\boldsymbol{d}=(0,0,1)^{T}$ and $\boldsymbol{p}=(1,0,0)^{T}$. We fix $\kappa_{s}=16 \pi$ and $R=1$. We observe
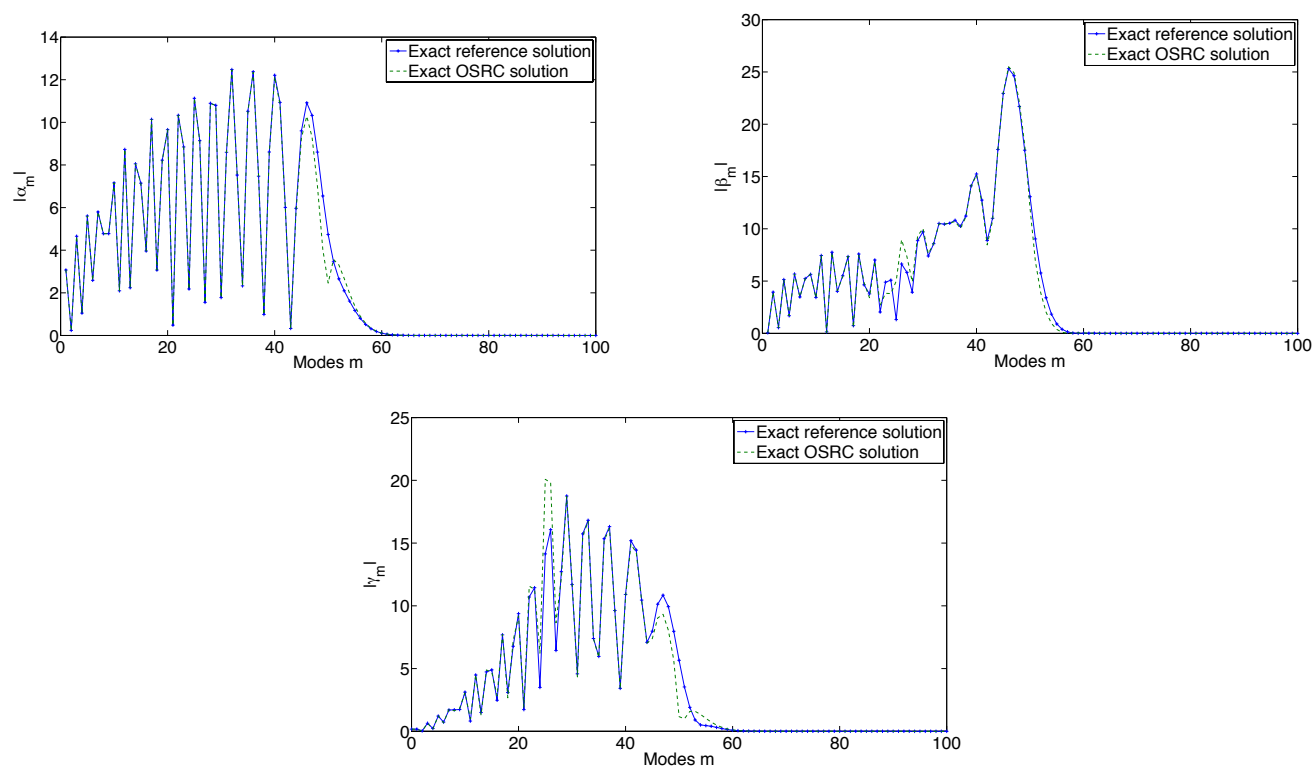

Figure 1: Diffraction of incident plane S-waves by a unit sphere $\left(\kappa_{s}=16 \pi\right)$ : modulus of the coefficients $\alpha_{m}^{\text {ex }}$ and $\alpha_{m}^{\mathrm{app}}$ (left), $\beta_{m}^{\mathrm{ex}}$ and $\beta_{m}^{\text {app }}$ (center), $\gamma_{m}^{\text {ex }}$ and $\gamma_{m}^{\text {app }}$ (right).

a very good approximation of the exact coefficients for all the modes, except in the transition zones for $m \approx \kappa_{p}$ and $m \approx \kappa_{s}$. These localized errors are due to the singularity that arises in the square-roots in (17) and (18). A solution to regularize the square-root operator consists in adding a small artificial damping parameter to the wavenumbers $\kappa_{p}$ and $\kappa_{s}$. We set $\kappa_{\alpha, \varepsilon}=\kappa_{\alpha}+i \varepsilon_{\alpha}$, with $\varepsilon_{\alpha}>0, \alpha=p, s$. The new approximate DtN operator is written

$$
\tilde{\boldsymbol{t}}_{\mid \Gamma}=\left(\left(\mathrm{I}+\boldsymbol{\Lambda}_{\mathbf{2}, \varepsilon}\right)^{-1} \boldsymbol{\Lambda}_{\mathbf{1}, \varepsilon}+2 \mu \mathcal{M}\right) \boldsymbol{u}_{\mid \Gamma}
$$

with

$$
\boldsymbol{\Lambda}_{\mathbf{1}, \varepsilon}=i \rho \omega^{2}\left[\boldsymbol{n}\left(\Delta_{\Gamma}+\kappa_{p, \varepsilon}^{2} \mathrm{I}\right)^{-\frac{1}{2}} \boldsymbol{n} \cdot \mathrm{I}_{\boldsymbol{n}}+\left(\boldsymbol{\Delta}_{\Gamma}+\kappa_{s, \varepsilon}^{2} \mathrm{I}_{\boldsymbol{t}}\right)^{-\frac{1}{2}}\left(\mathrm{I}_{\boldsymbol{t}}-\frac{1}{\kappa_{s, \varepsilon}^{2}} \operatorname{curl}_{\Gamma} \operatorname{curl}_{\Gamma}\right)\right]
$$

and

$$
\boldsymbol{\Lambda}_{\mathbf{2}, \varepsilon}=-i\left(\boldsymbol{\nabla}_{\Gamma}\left(\Delta_{\Gamma}+\kappa_{s, \varepsilon}^{2} \mathrm{I}\right)^{-\frac{1}{2}} \boldsymbol{n} \cdot \mathrm{I}_{\boldsymbol{n}}-\boldsymbol{n}\left(\Delta_{\Gamma}+\kappa_{p, \varepsilon}^{2} \mathrm{I}\right)^{-\frac{1}{2}} \operatorname{div}_{\Gamma} \mathrm{I}_{\boldsymbol{t}}\right)
$$

The aim is now to choose the damping parameters in order to minimize the errors between the exact and approximate coefficients in the transition zone. Previous works in acoustics and electromagnetism have exhibited an optimal damping parameter by solving an optimization problem for a sufficiently large wavenumber. In view of Proposition 3.2 it seems natural to consider the same expression of the optimal parameters for elastodynamic problems as for the Helmholtz [29, 7 ] and Maxwell [33] exterior problems, given respectively by $\varepsilon_{p, A}=0.39 \kappa_{p}^{1 / 3}\left(\mathcal{H}^{2}\right)^{1 / 3}$ and $\varepsilon_{s, E M}=$ $0.39 \kappa_{s}^{1 / 3}\left(\mathcal{H}^{2}\right)^{1 / 3}$, where $\mathcal{H}$ is the mean curvature of the boundary $\Gamma$. This choice can also be justified by the observation that the expression of the coefficient $\alpha_{m}^{\text {ex }}$ is the same as in the Maxwell case so that the optimization of $\alpha_{m}^{\text {app }}$ would give exactely $\varepsilon_{s, E M}$. Moreover, we have numerically observed that this choice improves the accuracy of the approximate DtN (27). We report on Figure 2 the exact and approximate coefficients. The accuracy is better in the zones $m \approx \kappa_{p}$ and $m \approx \kappa_{s}$ when considering the damping parameters $\varepsilon_{p, A}$ and $\varepsilon_{s, E M}$ without deteriorating the 
approximation for the propagative $\left(m \ll \kappa_{p}\right)$ and evanescent modes $\left(m \gg \kappa_{s}\right)$. We consider these two parameters in the sequel of the paper.
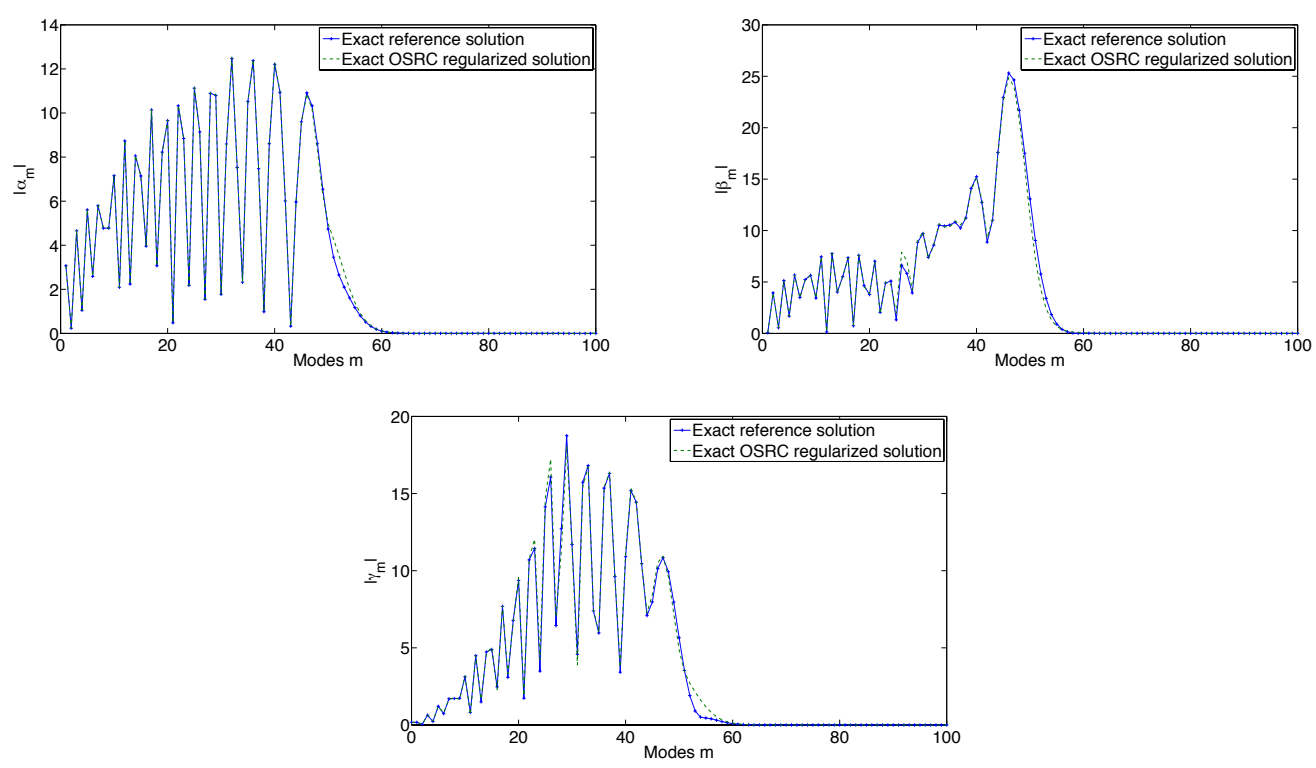

Figure 2: Diffraction of incident plane S-waves by a unit sphere $\left(\kappa_{s}=16 \pi\right)$ : modulus of the coefficients $\alpha_{m}^{\mathrm{ex}}$ and $\alpha_{m, \varepsilon}^{\mathrm{app}}$ (left), $\beta_{m}^{\mathrm{ex}}$ and $\beta_{m, \varepsilon}^{\mathrm{app}}$ (center), $\gamma_{m}^{\mathrm{ex}}$ and $\gamma_{m, \varepsilon}^{\mathrm{app}}$ (right).

\subsection{Local approximate DtN map}

A crucial point is to propose a local representation of the square-root operators involved in 28 and 29). To this end, we use complex rational Padé approximants of order $2 L+1$ with a rotating branch-cut technique of angle $\theta[8,48,52$, 17: for $z \in \mathbb{C}$, one has

$$
(1+z)^{-1 / 2} \approx \frac{P_{L}(z)}{Q_{L}(z)}=r_{0}+\sum_{\ell=1}^{L} \frac{r_{\ell}}{z-q_{\ell}}
$$

where $P_{L}$ and $Q_{L}$ are two polynomials of degree $L$. The complex coefficients $r_{\ell}, \ell=0, \ldots, L$, and $q_{\ell}, \ell=1, \ldots, L$ depend on the angle $\theta$. The notation $z^{1 / 2}$ designates the principal determination of the square-root of a complex number $z$ with branch-cut along the negative real axis. The use of Padé approximants leads to the following relation on a general surface $\Gamma$

$$
\tilde{\boldsymbol{t}}_{\mid \Gamma}^{\text {Pade }}=\left(\left(\mathrm{I}+\widetilde{\boldsymbol{\Lambda}}_{\mathbf{2}, \varepsilon}\right)^{-1} \widetilde{\boldsymbol{\Lambda}}_{\mathbf{1}, \varepsilon}+2 \mu \mathcal{M}\right) \boldsymbol{u}_{\mid \Gamma} .
$$

The local representations of the operators $\boldsymbol{\Lambda}_{\mathbf{1}, \varepsilon}$ and $\boldsymbol{\Lambda}_{\mathbf{2}, \varepsilon}$ are given respectively by

$$
\begin{aligned}
\widetilde{\boldsymbol{\Lambda}}_{\mathbf{1}, \varepsilon} & =i \rho \omega^{2}\left[\frac{1}{\kappa_{p, \varepsilon}} \boldsymbol{n}\left(r_{0} \mathrm{I}+\sum_{\ell=1}^{L} r_{\ell}\left(\frac{\Delta_{\Gamma}}{\kappa_{p, \varepsilon}^{2}}-q_{\ell} \mathrm{I}\right)^{-1}\right) \boldsymbol{n} \cdot \mathrm{I}_{\boldsymbol{n}}\right. \\
& \left.+\frac{1}{\kappa_{s, \varepsilon}}\left(r_{0} \mathrm{I}+\sum_{\ell=1}^{L} r_{\ell}\left(\mathbf{L}-q_{\ell} \mathrm{I}\right)^{-1}\right)\left(\mathrm{I}_{t}-\operatorname{curl}_{\Gamma} \frac{1}{\kappa_{s, \varepsilon}^{2}} \operatorname{curl}_{\Gamma}\right)\right] \text { and }
\end{aligned}
$$




$$
\begin{aligned}
\widetilde{\boldsymbol{\Lambda}}_{\mathbf{2}, \varepsilon} & =i\left[-\frac{1}{\kappa_{s, \varepsilon}} \nabla_{\Gamma}\left(r_{0} \mathrm{I}+\sum_{\ell=1}^{L} r_{\ell}\left(\frac{\Delta_{\Gamma}}{\kappa_{s, \varepsilon}^{2}}-q_{\ell} \mathrm{I}\right)^{-1}\right) \boldsymbol{n} \cdot \mathrm{I}_{\boldsymbol{n}}\right. \\
& \left.+\frac{1}{\kappa_{p, \varepsilon}} \boldsymbol{n}\left(r_{0} \mathrm{I}+\sum_{\ell=1}^{L} r_{\ell}\left(\frac{\Delta_{\Gamma}}{\kappa_{p, \varepsilon}^{2}}-q_{\ell} \mathrm{I}\right)^{-1}\right) \operatorname{div}_{\Gamma} \mathrm{I}_{\boldsymbol{t}}\right]
\end{aligned}
$$

where we have set $\mathbf{L}:=\nabla_{\Gamma} \frac{1}{\kappa_{s, \varepsilon}^{2}} \operatorname{div}_{\Gamma}-\operatorname{curl}_{\Gamma} \frac{1}{\kappa_{s, \varepsilon}^{2}} \operatorname{curl}_{\Gamma}$ in view of the decomposition [54, Equation (2.5.192)] of the operator $\boldsymbol{\Delta}_{\Gamma}$ acting on tangent vector fields. We compute the analytical expression of the solution $\tilde{\boldsymbol{t}}_{\mid \Gamma}^{\text {Pade }}$ for the scattering by the sphere $\mathbf{S}^{2}$

$$
\tilde{\boldsymbol{t}}_{\mid \Gamma}^{\text {Pade }}(\boldsymbol{x})=\sum_{m=1}^{\infty} \sum_{j=-m}^{m}\left(\alpha_{m}^{\text {Pade }} \operatorname{curl}_{\mathbf{S}^{2}} Y_{m, j}(\hat{\boldsymbol{x}})+\beta_{m}^{\text {Pade }} \nabla_{\mathbf{S}^{2}} Y_{m, j}(\hat{\boldsymbol{x}})\right)+\sum_{m=0}^{\infty} \sum_{j=-m}^{m} \gamma_{m}^{\text {Pade }} \hat{\boldsymbol{x}} Y_{m, j}(\hat{\boldsymbol{x}}) .
$$

The coefficients depend on the Padé parameters: the angle of rotation $\theta$ and the order $2 L+1$. We report in Table 1 the error $\left\|\alpha^{\text {app }}-\alpha^{\text {Pade }}\right\|$ according to $\theta$ and $L$, with $\alpha^{\text {app }}=\left(\alpha_{m}^{\text {app }}\right)_{1 \leq m \leq m_{\max }}$, $\alpha^{\text {Pade }}=\left(\alpha_{m}^{\text {Pade }}\right)_{1 \leq m \leq m_{\max }}$, and $\|\cdot\|$ the Euclidian norm. We consider the scattering by a unit sphere of an incident plane $S$-wave with $\boldsymbol{d}=(0,0,1)^{T}$ and $\boldsymbol{p}=(1,0,0)^{T}$. We fix $\kappa_{s}=6 \pi$ and $m_{\max }=3\left[\kappa_{s}\right]$, where $[\cdot]$ denotes the integer part of a real number. As expected, the increase of the Padé order $N$ leads to better accuracy. An appropriate angle seems to be $\theta=\pi / 2$. We obtain similar results for the other coefficients. From now on, we fix $\theta=\pi / 2$.

Table 1: Diffraction of an incident plane S-wave by a unit sphere: $\left\|\alpha^{\text {app }}-\alpha^{\text {Pade }}\right\|$ vs $\theta$ and $N$.

\begin{tabular}{c|ccc}
\hline$\theta$ & $N=4$ & $N=8$ & $N=12$ \\
\hline 0 & $1.05 \mathrm{e} 00$ & $2.22 \mathrm{e}-01$ & $7.35 \mathrm{e}-02$ \\
$\pi / 4$ & $5.71 \mathrm{e}-02$ & $6.4 \mathrm{e}-04$ & $1.42 \mathrm{e}-05$ \\
$\pi / 3$ & $2.85 \mathrm{e}-02$ & $1.48 \mathrm{e}-04$ & $1.59 \mathrm{e}-06$ \\
$\pi / 2$ & $2.99 \mathrm{e}-02$ & $8.64 \mathrm{e}-05$ & $5.94 \mathrm{e}-07$ \\
$2 \pi / 3$ & $2.49 \mathrm{e}-01$ & $2.74 \mathrm{e}-03$ & $6.92 \mathrm{e}-04$ \\
\hline
\end{tabular}

\section{An application of the approximate local DtN: the OSRC method}

\subsection{Principle of the OSRC method}

One possible application of the approximate DtN (31) is as an OSRC [3, 33, 44, to solve approximately the exterior Navier problem (1)-(3)-(4). The method consists in considering the boundary differential equation

$$
\tilde{\boldsymbol{t}}_{\mid \Gamma}=-\left(\left(\mathrm{I}+\widetilde{\boldsymbol{\Lambda}}_{\mathbf{2}, \varepsilon}\right)^{-1} \widetilde{\boldsymbol{\Lambda}}_{\mathbf{1}, \varepsilon}+2 \mu \mathcal{M}\right) \boldsymbol{u}_{\mid \Gamma}^{\text {inc }}
$$

directly on the physical surface $\Gamma$. The approximation of the DtN map is evaluated by solving a set of local differential equations posed on $\Gamma$ (i.e. on the boundary similarly to BEMs). The solution of the sparse linear systems (contrary to BEMs that lead to dense systems) associated with the discretization of (34) gives an approximation of the trace $\tilde{\boldsymbol{t}}_{\mid \Gamma}$, and hence of the scattered field

$$
\boldsymbol{u}(\boldsymbol{x}) \approx-\mathcal{D} \boldsymbol{u}_{\mid \Gamma}^{\text {inc }}(\boldsymbol{x})-\mathcal{S} \tilde{\boldsymbol{t}}_{\mid \Gamma}(\boldsymbol{x}), \quad \boldsymbol{x} \in \Omega^{+} .
$$

Similarly, the approximate far-field pattern is computed via the integral representation formula

$$
\boldsymbol{u}^{\infty}(\hat{\boldsymbol{x}}) \approx-\mathscr{F}_{\mathrm{D}} \boldsymbol{u}_{\mid \Gamma}^{\mathrm{inc}}(\hat{\boldsymbol{x}})-\mathscr{F}_{\mathrm{S}} \tilde{\boldsymbol{t}}_{\mid \Gamma}(\hat{\boldsymbol{x}})
$$

where the far-field operators $\mathscr{F}_{\mathrm{S}}$ and $\mathscr{F}_{\mathrm{D}}$ are defined by 11$]-(12)$. The evaluation of the approximate Neumann trace $\tilde{t}_{\mid \Gamma}$ is decomposed into the following three steps: 
Step 1: Compute the intermediate variable $\boldsymbol{v} \in \boldsymbol{H}^{\frac{1}{2}}(\Gamma)$ such that

$$
\boldsymbol{v}=-\widetilde{\boldsymbol{\Lambda}}_{\mathbf{1}, \varepsilon} \boldsymbol{u}_{\mid \Gamma}^{\mathrm{inc}}
$$

Step 2: Solve the boundary differential equation: find $\boldsymbol{q} \in \boldsymbol{H}^{-\frac{1}{2}}(\Gamma)$ solution of

$$
\left(\mathrm{I}+\widetilde{\boldsymbol{\Lambda}}_{\mathbf{2}, \varepsilon}\right) \boldsymbol{q}=\boldsymbol{v}
$$

Step 3: Deduce an approximation of the Neumann trace $\tilde{\boldsymbol{t}}_{\mid \Gamma} \in \boldsymbol{H}^{-\frac{1}{2}}(\Gamma)$

$$
\tilde{\boldsymbol{t}}_{\mid \Gamma}=\boldsymbol{q}-2 \mu \mathcal{M} \boldsymbol{u}_{\mid \Gamma}^{\mathrm{inc}}
$$

The treatment of the three steps involves only sparse matrices. We point out that the second one requires to solve a linear system (using an iterative solver) to find the intermediate variable $\boldsymbol{q}$. The efficiency of the OSRC method is obtained at the expense of the accuracy. Thus it is less accurate than integral equation methods but it is extremely faster. Its numerical efficiency is related to the (limited) level of accuracy required in the approximate DtN operator. In Section 5.3 . we demonstrate on numerical examples that the OSRC method is nevertheless a very interesting tool to analyze high frequency scattering phenomena.

\subsection{Finite element discretization}

We consider a triangulation of $\Gamma$ with $N_{T}$ triangular elements and $N_{V}$ vertices. The polyedric interpolated surface is denoted by $\Gamma_{h}$. For the discretization, we use classical $\mathbb{P}_{1}$ boundary finite elements. We set $V_{h}=\mathbb{P}_{1}\left(\Gamma_{h}\right)$ and $\boldsymbol{V}_{h}=V_{h}^{3}$ with $\operatorname{dim} \boldsymbol{V}_{h}=3 N_{V}$. For example, the $\mathbb{P}_{1}$-interpolation $\boldsymbol{g}_{h}:=\left(\boldsymbol{u}_{\mid \Gamma}^{\mathrm{inc}}\right)_{h}$ of the Dirichlet data is given by

$$
\boldsymbol{g}_{h}(\boldsymbol{x})=\sum_{i=1}^{3} \sum_{j=1}^{N_{V}} g_{i}\left(\boldsymbol{a}_{j}\right) \varphi_{j}(\boldsymbol{x}) \boldsymbol{e}_{i}, \quad \boldsymbol{x} \in \Gamma_{h},
$$

where $\boldsymbol{a}_{j}, 1 \leq j \leq N_{V}$, are the vertices of the mesh and the functions $\varphi_{j}, 1 \leq j \leq N_{V}$, the $\mathbb{P}_{1}$ basis functions on $\Gamma_{h}$. We use the standard scalar product given by

$$
(\boldsymbol{u}, \boldsymbol{v}):=\int_{\Gamma_{h}} \boldsymbol{u} \cdot \boldsymbol{v} d \Gamma_{h}
$$

The discretization of the OSRC $(34)$ is decomposed into three steps:

Step 1: The discrete weak formulation for the application of the operator $\widetilde{\Lambda}_{1, \varepsilon}$ (eq. 37p) is given in the following algorithm:

(a) Knowing the incident field, solve the $L$ uncoupled equations to find $v_{h}^{\ell} \in V_{h}$ such that

$$
\left(\kappa_{p, \varepsilon_{h}}^{-1} \nabla_{\Gamma_{h}} v_{h}^{\ell}, \kappa_{p, \varepsilon_{h}}^{-1} \nabla_{\Gamma_{h}} v_{h}^{\prime}\right)+q_{\ell}\left(v_{h}^{\ell}, v_{h}^{\prime}\right)=-\left(\boldsymbol{n}_{h} \cdot \boldsymbol{g}_{h}, v_{h}^{\prime}\right), \quad v_{h}^{\prime} \in V_{h}, \quad \ell=1, \ldots, L .
$$

Note that the coefficients $r_{0}, r_{\ell}, q_{\ell}, \ell=1, \ldots, L$, are determined by the Padé approximation 30 .

(b) Evaluate the intermediate variable $z_{h} \in V_{h}$ such that

$$
\left(\kappa_{p, \varepsilon_{h}} z_{h}, z_{h}^{\prime}\right)=r_{0}\left(\boldsymbol{n}_{h} \cdot \boldsymbol{g}_{h}, z_{h}^{\prime}\right)+\sum_{\ell=1}^{L} r_{\ell}\left(v_{h}^{\ell}, z_{h}^{\prime}\right), \quad z_{h}^{\prime} \in V_{h} .
$$

(c) Independently from steps (a)-(b), compute $\boldsymbol{b}_{h} \in \boldsymbol{V}_{h}$ solution to

$$
\left(\boldsymbol{b}_{h}, \boldsymbol{b}_{h}^{\prime}\right)=\left(\boldsymbol{n}_{h} \times\left(\boldsymbol{g}_{h} \times \boldsymbol{n}_{h}\right), \boldsymbol{b}_{h}^{\prime}\right)-\left(\kappa_{s, \varepsilon_{h}}^{-1} \operatorname{curl}_{\Gamma_{h}} \boldsymbol{g}_{h}, \kappa_{s, \varepsilon_{h}}^{-1} \operatorname{curl}_{\Gamma_{h}} \boldsymbol{b}_{h}^{\prime}\right), \quad \boldsymbol{b}_{h}^{\prime} \in \boldsymbol{V}_{h} .
$$


(d) For all $\left(\mathbf{u}_{h}^{\prime}, \rho_{h}^{\prime}\right) \in \boldsymbol{V}_{h} \times V_{h}$, solve the $L$ uncoupled systems of two equations

$$
\left\{\begin{array}{l}
q_{\ell}\left(\mathbf{u}_{h}^{\ell}, \mathbf{u}_{h}^{\prime}\right)+\left(\kappa_{s, \varepsilon_{h}}^{-1} \operatorname{curl}_{\Gamma_{h}} \mathbf{u}_{h}^{\ell}, \kappa_{s, \varepsilon_{h}}^{-1} \operatorname{curl}_{\Gamma_{h}} \mathbf{u}_{h}^{\prime}\right)-\left(\nabla_{\Gamma_{h}} \rho_{h}^{\ell}, \mathbf{u}_{h}^{\prime}\right)=-\left(\boldsymbol{b}_{h}, \mathbf{u}_{h}^{\prime}\right), \quad \ell=1, \ldots, L, \\
\left(\rho_{h}^{\ell}, \rho_{h}^{\prime}\right)+\left(\kappa_{s, \varepsilon_{h}}^{-1} \mathbf{u}_{h}^{\ell}, \kappa_{s, \varepsilon_{h}}^{-1} \nabla_{\Gamma_{h}} \rho_{h}^{\prime}\right)=0, \quad \ell=1, \ldots, L .
\end{array}\right.
$$

(e) Evaluate the intermediate variable $\mathbf{c}_{h} \in \boldsymbol{V}_{h}$

$$
\left(\kappa_{s, \varepsilon_{h}} \mathbf{c}_{h}, \mathbf{c}_{h}^{\prime}\right)=r_{0}\left(\boldsymbol{b}_{h}, \mathbf{c}_{h}^{\prime}\right)+\sum_{\ell=1}^{L} r_{\ell}\left(\mathbf{u}_{h}^{\ell}, \mathbf{c}_{h}^{\prime}\right), \quad \mathbf{c}_{h}^{\prime} \in \boldsymbol{V}_{h}
$$

(f) The variable $\boldsymbol{v}_{h} \in \boldsymbol{V}_{h}$ is finally given by

$$
\left(\boldsymbol{v}_{h}, \boldsymbol{v}_{h}^{\prime}\right)=-i \rho \omega^{2}\left(\left(z_{h}, \boldsymbol{n}_{h} \cdot \boldsymbol{v}_{h}^{\prime}\right)+\left(\mathbf{c}_{h}, \boldsymbol{v}_{h}^{\prime}\right)\right), \quad \boldsymbol{v}_{h}^{\prime} \in \boldsymbol{V}_{h}
$$

The discrete wavenumbers are expressed by $\kappa_{\alpha, \varepsilon_{h}}=\kappa_{s}+i \varepsilon_{h}$ with $\varepsilon_{h}=\beta_{\alpha} \kappa_{\alpha}^{1 / 3}\left(\mathcal{H}_{h}^{2}\right)^{1 / 3}$ $(\alpha=s, p)$. The quantity $\mathcal{H}_{h}$ is a piecewise constant interpolation of the mean curvature $\mathcal{H}$ over $\Gamma_{h}$ on each triangle of the triangulation. The numerical evaluation of $\mathcal{H}_{h}$ comes from the relation

$$
\mathcal{H}_{h}(\boldsymbol{x})=\frac{1}{2} \operatorname{div}_{\Gamma_{h}} \boldsymbol{n}_{h}(\boldsymbol{x})=\frac{1}{2} \sum_{k=1}^{3} \sum_{j=1}^{N_{V}}\left(\boldsymbol{n}_{h}\left(\boldsymbol{a}_{j}\right) \cdot \boldsymbol{e}_{k}\right)\left(\boldsymbol{e}_{k} \cdot \nabla_{\Gamma_{h}} \varphi_{j}(\boldsymbol{x})\right), \quad \boldsymbol{x} \in \Gamma_{h}
$$

Step 2: The solution $\mathbf{q}_{h} \in \boldsymbol{V}_{h}$ of the linear system 38 is obtained with the iterative solver GMRES. At each iteration, the matrix-vector product required by GMRES

$$
\left(\mathrm{I}+\widetilde{\Lambda}_{\mathbf{2}, \varepsilon}\right) \mathbf{x}=\mathbf{z}
$$

is realized by the following algorithm:

(a) Solve the $L$ uncoupled systems to find $v_{h}^{\ell} \in V_{h}$, such that

$$
\left(\kappa_{s, \varepsilon_{h}}^{-1} \nabla_{\Gamma_{h}} v_{h}^{\ell}, \kappa_{s, \varepsilon_{h}}^{-1} \nabla_{\Gamma_{h}} v_{h}^{\prime}\right)+q_{\ell}\left(v_{h}^{\ell}, v_{h}^{\prime}\right)=-\left(\boldsymbol{n}_{h} \cdot \mathbf{x}_{h}, v_{h}^{\prime}\right), \quad v_{h}^{\prime} \in V_{h}, \quad \ell=1, \ldots, L .
$$

(b) Evaluate the auxiliary variable $w_{h} \in V_{h}$

$$
\left(\kappa_{s, \varepsilon_{h}} w_{h}, w_{h}^{\prime}\right)=r_{0}\left(\boldsymbol{n}_{h} \cdot \mathbf{x}_{h}, w_{h}^{\prime}\right)+\sum_{\ell=1}^{L} r_{\ell}\left(v_{h}^{\ell}, w_{h}^{\prime}\right), \quad w_{h}^{\prime} \in V_{h}
$$

(c) Independently from steps (a)-(b), solve the $L$ uncoupled systems to find $u_{h}^{\ell} \in V_{h}$ such that

$$
\left(\kappa_{p, \varepsilon_{h}}^{-1} \nabla_{\Gamma_{h}} u_{h}^{\ell}, \kappa_{p, \varepsilon_{h}}^{-1} \nabla_{\Gamma_{h}} u_{h}^{\prime}\right)+q_{\ell}\left(u_{h}^{\ell}, u_{h}^{\prime}\right)=-\left(\operatorname{div}_{\Gamma_{h}} \mathrm{I}_{t} \mathbf{x}_{h}, u_{h}^{\prime}\right), \quad u_{h}^{\prime} \in V_{h}, \quad \ell=1, \ldots, L .
$$

(d) Evaluate the intermediate variable $y_{h} \in V_{h}$ solution to

$$
\left(\kappa_{p, \varepsilon_{h}} y_{h}, y_{h}^{\prime}\right)=r_{0}\left(\operatorname{div}_{\Gamma_{h}} \mathrm{I}_{t} \mathbf{x}_{h}, y_{h}^{\prime}\right)+\sum_{\ell=1}^{L} r_{\ell}\left(u_{h}^{\ell}, y_{h}^{\prime}\right), \quad y_{h}^{\prime} \in V_{h} .
$$

(e) The variable $\mathbf{z}_{h} \in \boldsymbol{V}_{h}$ is finally given by

$$
\left(\mathbf{z}_{h}, \mathbf{z}_{h}^{\prime}\right)=\left(\mathbf{x}_{h}, \mathbf{z}_{h}^{\prime}\right)+i\left(-\left(\nabla_{\Gamma_{h}} w_{h}, \mathbf{z}_{h}^{\prime}\right)+\left(y_{h}, \boldsymbol{n}_{h} \cdot \mathbf{z}_{h}^{\prime}\right)\right), \quad \mathbf{z}_{h}^{\prime} \in \boldsymbol{V}_{h}
$$

Step 3: The approximation $\boldsymbol{t}_{h}$ of the Neumann trace of the scattered field is finally obtained: Find 
$\boldsymbol{t}_{h} \in \boldsymbol{V}_{h}$ such that for all $\boldsymbol{t}_{h}^{\prime} \in \boldsymbol{V}_{h}$

$$
\left(\boldsymbol{t}_{h}, \boldsymbol{t}_{h}^{\prime}\right)=\left(\boldsymbol{q}_{h}, \boldsymbol{t}_{h}^{\prime}\right)-2 \mu\left(\mathcal{M}_{h} \boldsymbol{g}_{h}, \boldsymbol{t}_{h}^{\prime}\right)
$$

where $\boldsymbol{q}_{h}$ is computed in Step $\mathbf{2}$ and $\boldsymbol{\mathcal { M }}_{h}$ is the numerical evaluation of the tangential Günter derivative.

According to the decomposition of the DtN operator mentioned in Section 3.2 the application of the approximate acoustic NtD operator [7] and of the approximate MtE operator [33] appear in Step 1 and Step 2. The implementation is easy and only requires sparse matrices. The solution of the $4 L$ uncoupled (and thus embarrassingly parallel) sparse linear systems with $L$ small (typically $L=4$ ) is efficiently realized by a direct solver.

\subsection{Numerical efficiency of the OSRC for 3D elastodynamics}

We study the scattering of incident elastic plane waves of the form 25 by different obstacles. We compute the Neumann trace of the scattered field $\boldsymbol{u}$ (35) and the bistatic Scattering Cross Section (SCS) [2]:

$$
\operatorname{SCS}(\theta, \phi)=10 \log _{10}\left(4 \pi\left|\boldsymbol{u}^{\infty}(\hat{\boldsymbol{x}}(\theta, \phi))\right|^{2}\right) \quad(\mathrm{dB}),
$$

where the points $\hat{\boldsymbol{x}} \in \mathbf{S}^{2}$ are defined by $\hat{\boldsymbol{x}}:=\hat{\boldsymbol{x}}(\theta, \phi)=(\sin (\theta) \cos (\phi), \sin (\theta) \sin (\phi), \cos (\theta))$. We consider several geometries. First, a unit sphere is used for validation purposes by comparison with the analytical solutions A.1 and A.2. The second object is an ellipsoid with semi-axes $a=1, b=0.5$ and $c=b$ along the $x$-, $y$ - and $z$-directions respectively (ellipsoid $a b b$ ), or with semi-axes $a=1, b=0.75$ and $c=0.5$ (ellipsoid $a b c$ ). The last geometry is a trapping domain. We consider a semi-concave sphere (Sshape) (Fig. 3). The number of vertices of the different meshes used are given in Table 2. We denote by $n_{\lambda_{s}}=\lambda_{s} / h$ the density of discretization points per S-wavelength where $h$ is the average length of the edges of the triangles. The simulations are performed with Matlab. All the tests are run on an Dell PowerEdge R900 with 4 chips Intel Xeon E7 (10 cores, $2 \mathrm{GHz})$ and $1 \mathrm{~Tb}$ of RAM.

Table 2: Number of vertices for the different meshes used in this Section.
\begin{tabular}{c|cccccc} 
Mesh & sphere 3 & sphere 4 & ellipsoid abb & ellipsoid abc & Sshape \\
$N_{V}$ & 40962 & 61033 & 40962 & 40064 & 61542 \\
\hline
\end{tabular}

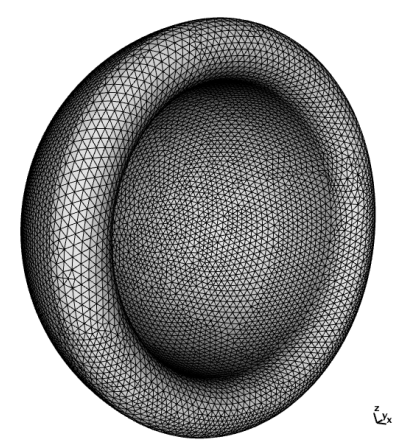

Figure 3: Geometry of the semi-concave sphere.

The efficiency and accuracy of the method depend on different parameters: the damping parameters $\varepsilon_{s, p}$, the order $2 L+1$ and the angle $\theta$ of the Padé approximation and the stopping criterion of the GMRES solver (without restart) in the second step of the OSRC method. We summarize the parameters used for all the following simulations (unless indicated otherwise) in Table 3. The choice of the regularizing parameters $\varepsilon_{s, p}$ and the ones used in the localization 
process has been discussed in the previous Sections 4.1 and 4.2 . Concerning the tolerance of the GMRES, note that the OSRC method is an approximate method with a lower accuracy than the reference method of boundary integral equations. As a result, it is sufficient to fix the tolerance in the GMRES stopping criterion to $10^{-3}$. For this choice, the convergence of the GMRES is achieved within a small number of iterations.

Table 3: Important Parameters of the OSRC and corresponding fixed values in the remaining of the Article.

\begin{tabular}{c|ccccc}
\hline Parameters & $\varepsilon_{s}$ & $\varepsilon_{p}$ & $L$ & $\theta$ & tol \\
Values & $0.4 \kappa_{s}^{1 / 3}\left(\mathcal{H}^{2}\right)^{1 / 3}$ & $0.4 \kappa_{p}^{1 / 3}\left(\mathcal{H}^{2}\right)^{1 / 3}$ & 4 & $\pi / 2$ & $1 . \mathrm{e}-03$ \\
\hline
\end{tabular}

First, we study the convergence of the finite element method. We consider the scattering of incident plane $\mathrm{P}$-waves with $\boldsymbol{p}=\boldsymbol{d}=(0,0,1)^{T}$ or S-waves with $\boldsymbol{d}=(0,0,1)^{T}$ and $\boldsymbol{p}=(1,0,0)^{T}$ by a unit sphere. The bistatic SCS is evaluated at points $\hat{\boldsymbol{x}}=(\sin (\theta), 0, \cos (\theta))$ where $\theta \in[0,2 \pi]$, namely $\operatorname{SCS}(\theta, 0)$. We report in Table 4 the relative error between the analytical SCS relative to the OSRC formulation (cf. Appendix) and the computed SCS with respect to the discretization density $n_{\lambda_{\alpha}}$. These results attest both the convergence of the finite element approximation and the accuracy of the OSRC method. As expected, when decreasing the tolerance of GMRES to $10^{-3}$, the accuracy of the numerical solution is improved.

Table 4: Diffraction of incident plane P- or S-waves by a unit sphere: accuracy of the finite element method.

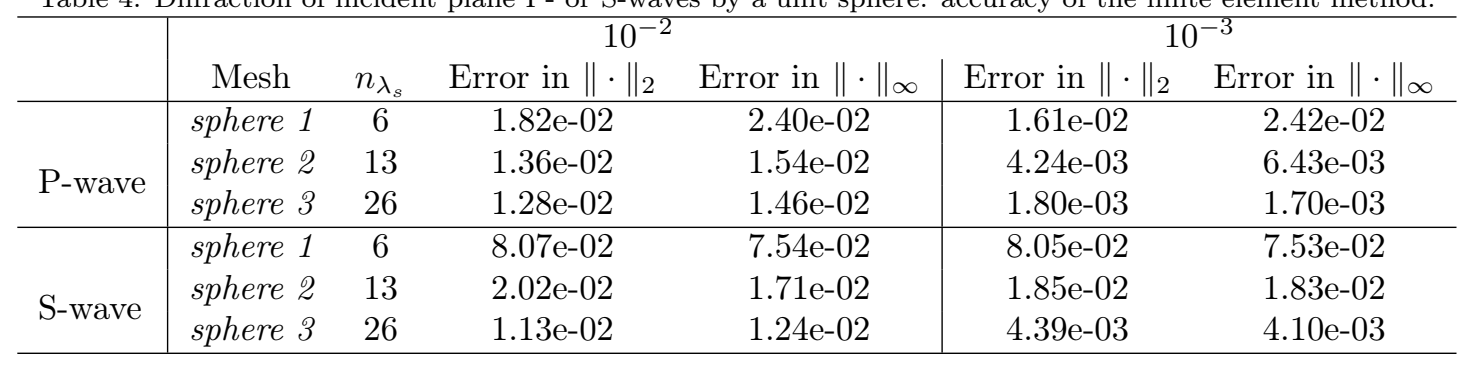

We check now the efficiency of the OSRC by considering first the ideal configuration of a unit sphere and incident plane P-waves with $\boldsymbol{p}=\boldsymbol{d}=(0,0,1)^{T}$, S-waves with $\boldsymbol{d}=(0,0,1)^{T}$ and $\boldsymbol{p}=(1,0,0)^{T}$ or a combination of $\mathrm{P}$ and $\mathrm{S}$ waves with $\boldsymbol{d}=(0,0,1)^{T}$ and $\boldsymbol{p}=1 / \sqrt{2}(1,0,1)^{T}$. The OSRC-based solution is compared to the analytical solution A.1 and the analytical OSRCbased solution A.2. The analytical OSRC-based solution represents the best solution that we can expect when using the OSRC. In Figure 4, the Neumann traces of the scattered field for $\kappa_{s}=8 \pi$ computed on the mesh sphere 3 (corresponding to $n_{\lambda_{s}}=13$ ) are reported. We observe a good agreement between the analytical and computed OSRC-based surface fields. In addition, the OSRC method allows a correct approximation of the reference exact solution. Similar conclusions hold when we check the accuracy of the $\operatorname{SCS}(\theta, 0)$ (cf. Figure 5). We also report the SCS for $\kappa_{s}=16 \pi$ with mesh sphere 4 (corresponding to $n_{\lambda_{s}}=8$ ). In that case, some oscillations appear in the OSRC solution. This is due to the small density of points per wavelength. However, the principal informations on the SCS are still well reproduced and a better accuracy is expected if a finer mesh is used. Finally, numerical results in Fig. 6] show the crucial role of the regularization process and confirm the study made in Section 4.1. The chosen regularization parameters lead to a very good approximation of the SCS for all angles while spurious oscillations are observed without damping, particularly in the zone of grazing rays.

We consider the scattering of plane waves by an ellipsoid (with $\kappa_{s}=8 \pi$ ) for two configurations described in Table 5. We report in Figure 7 the SCS computed with a spectral method [46] (considered as the reference one) and the SCS obtained with the OSRC method. Even for this more complex geometry of scatterer, the SCS is correctly reproduced for the two configurations. The only minor errors occur in the shadow zone.

The last obstacle is the semi concave sphere presented in Fig. 3. This trapping domain is known to be more difficult for the OSRC method [3, 33. The data are incident plane P-waves 


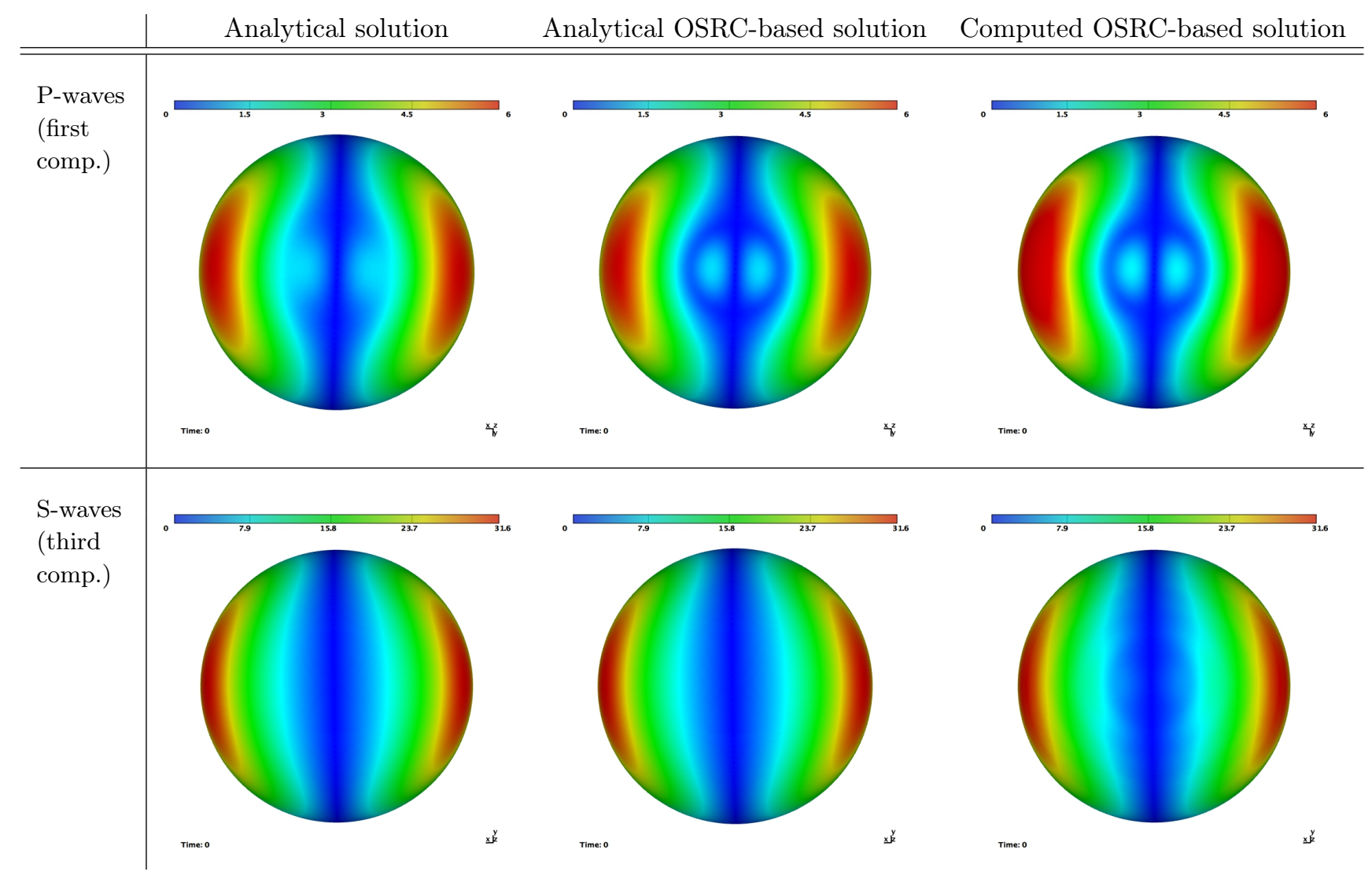

Figure 4: Diffraction of plane P-waves (first row) and S-waves (second row) by a unit sphere $\left(\kappa_{s}=8 \pi\right)$ : comparison of the Neumann traces of the scattered field (left: analytical solution, center: analytical OSRC-based solution and right: computed OSRC-based solution). For the P-wave (resp. S-wave) only the first component (resp. third component) is represented. 

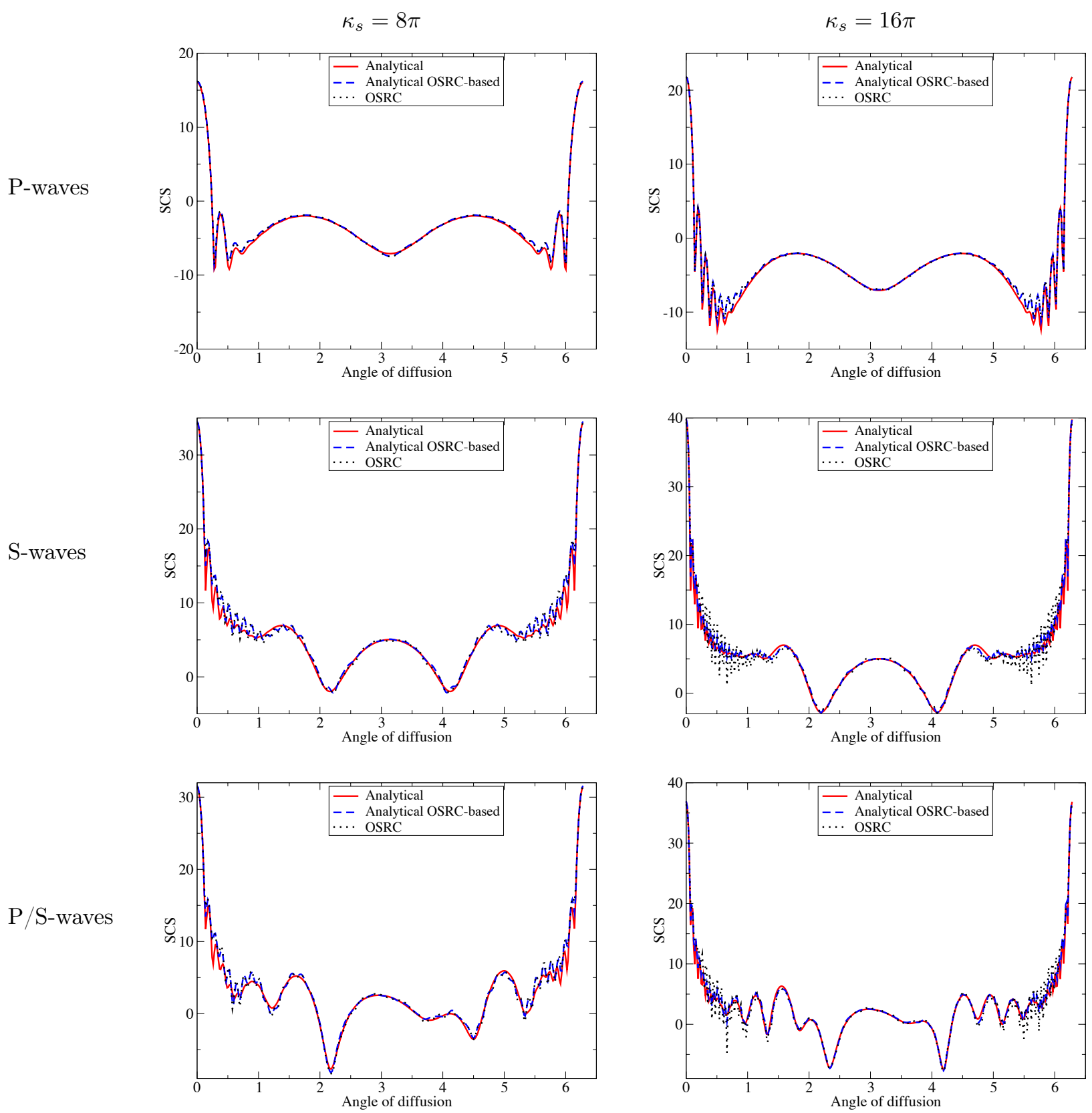

Figure 5: Diffraction of plane P-waves, S-waves and P/S-waves by a unit sphere (for $\kappa_{s}=8 \pi$ and $\left.\kappa_{s}=16 \pi\right)$ : comparison of the SCS for the analytical solution, the analytical OSRC-based solution and the OSRC-based solution.

Table 5: Description of the two tests used for the scattering by an ellipsoid.

\begin{tabular}{|c|c|c|c|c|c|}
\hline $\begin{array}{c}\text { Incident } \\
\text { waves }\end{array}$ & $d$ & $p$ & Geometry & $n_{\lambda_{s}}$ & SCS \\
\hline $\mathrm{P}$ & $(-1,0,0)^{T}$ & $\boldsymbol{p}=(1,0,0)^{T}$ & $a b c$ & 17 & $\begin{array}{c}\operatorname{SCS}(\pi / 2, \phi) \\
\phi \in[0,2 \pi]\end{array}$ \\
\hline $\mathrm{S}$ & $(0,0,-1)^{T}$ & $\boldsymbol{p}=(0,1,0)^{T}$ & $a b b$ & 19 & $\begin{array}{l}\operatorname{SCS}(\theta, 0) \\
\theta \in[0,2 \pi]\end{array}$ \\
\hline
\end{tabular}




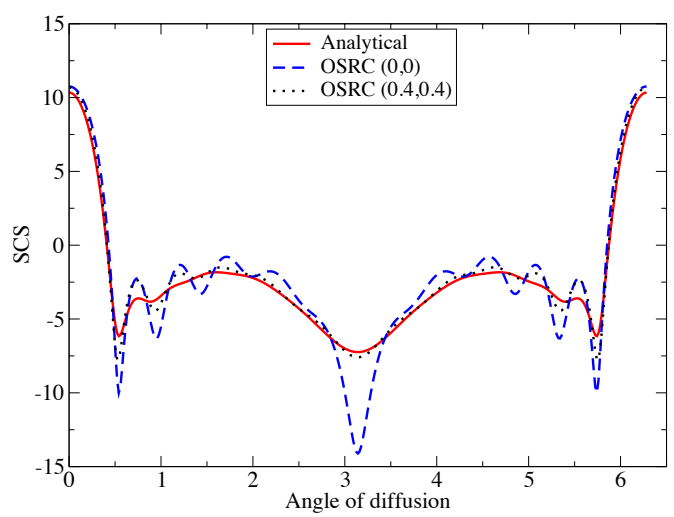

Figure 6: Diffraction of incident plane P-waves by a unit sphere $\left(\kappa_{s}=4 \pi\right)$ : study of the effect of the parameters $\varepsilon_{p}$ and $\varepsilon_{s}$. The SCS approximations if no regularization is added and if some regularization is added are compared with the analytic solution.

P-waves

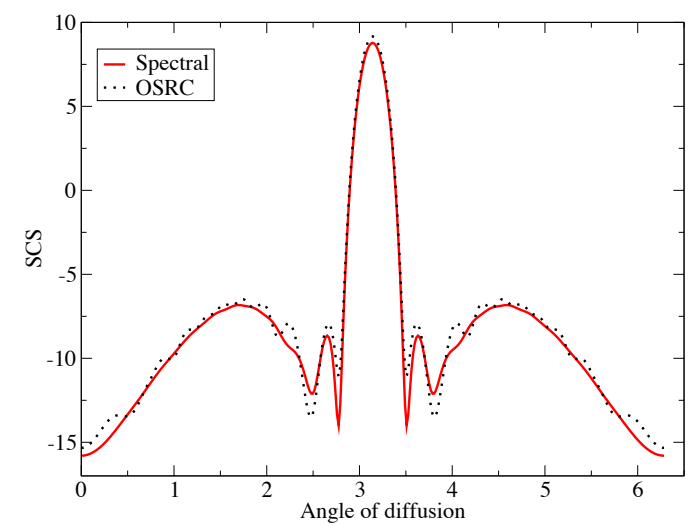

S-waves

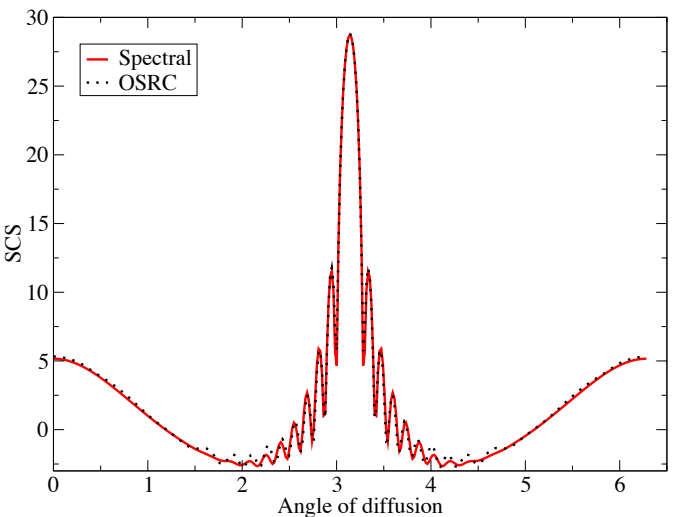

Figure 7: Diffraction of plane P-waves and S-waves by an ellipsoid $\left(\kappa_{s}=8 \pi\right)$ : comparison of the SCS for the OSRC-based solution and the spectral method-based solution.

with $\boldsymbol{p}=\boldsymbol{d}=(-1,0,0)^{T}$ and $\kappa_{s}=8 \pi$. This test corresponds to an incident wave that hits the concave part of the obstacle. The reference solution is computed using the FM-BEM [22]. We compute $\operatorname{SCS}(\theta, 0)$ with $\theta \in[0,2 \pi]$. We report in Fig. 8 the SCS. As expected, the accuracy on the SCS is better in the convex (sphere) part than in the cavity part.

\section{Conclusions and future work}

In this article, we have proposed a new class of approximate local Dirichlet-to-Neumann maps for three-dimensional elastodynamics. The methodology to construct and to evaluate these operators is detailed including regularization and localization steps. Furthermore, a physical interpretation is given to link these approximations to the ones obtained in acoustics and electromagnetism. A first application is presented in the context of the OSRC method. The approach has been numerically evaluated for many configurations (in terms of incident fields, scatterers or frequencies). Numerical simulations attest that the proposed approximate local DtN maps applied as OSRC operators reproduce in a fast and approximate way the near- and far-fields, especially when the scatterer is convex and the frequency is high. As expected, the OSRC performance is less satisfactory in the case of non-convex obstacles. 


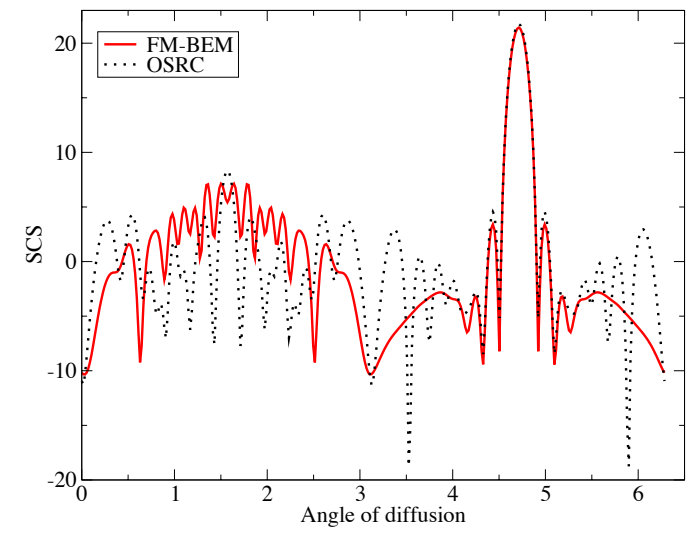

Figure 8: Diffraction of incident plane P-waves by a semi concave sphere $\left(\kappa_{s}=8 \pi\right)$.

Previous works in acoustics and electromagnetism show that this accuracy is nevertheless acceptable in other applications. And such approximate local DtN operators may play a central role in improving other numerical methods for wave propagation: to design inexpensive and efficient analytical preconditioners $([5,30,6,1,16,31,32,15])$ in the context of integral equations method, to be served as very good initial guesses for such iterative methods, to derive Absorbing Boundary Conditions for volume methods $([4,37,35,9,10])$ or to be used in the context of Domain Decomposition Methods ([36, 49, 14]). These extensions will be the subjects of promising forthcoming researches.

\section{Acknowledgments.}

The authors would like to thank the anonymous referees for useful comments and suggestions.

\section{A. Appendix}

In this appendix, we give the analytical expressions of the solution to the time-harmonic scattering problem of a plane wave by a rigid elastic sphere of radius $R>0$ and the approximate solution obtained using the OSRC method, in terms of the vector spherical harmonics.

Notations. We denote by $Y_{m, j}$ the spherical harmonics of order $m$ for $j=-m, \ldots, m$, with $m, j \in \mathbb{N}$ [54, Equation (2.4.78)]; by $j_{m}$ the spherical Bessel function of order $m \in \mathbb{N}$ and by $h_{m}^{(1)}$ the spherical Hankel function of the first kind and order $m \in \mathbb{N}$. We set $z_{m}^{(1)}=j_{m}$ and $z_{m}^{(3)}=$ $h_{m}^{(1)}$. We introduce the scalar function $u_{m, j}^{(k)}\left(\kappa_{p}, \boldsymbol{x}\right)=z_{m}^{(k)}\left(\kappa_{p}|\boldsymbol{x}|\right) Y_{m, j}(\hat{\boldsymbol{x}})$ and vector functions $M_{m, j}^{(k)}\left(\kappa_{s}, \boldsymbol{x}\right)=\operatorname{curl}\left(\boldsymbol{x} u_{m, j}^{(k)}\left(\kappa_{s}, \boldsymbol{x}\right)\right)$ and $N_{m, j}^{(k)}\left(\kappa_{s}, \boldsymbol{x}\right)=\frac{1}{i \kappa_{s}} \operatorname{curl} M_{m, j}^{(k)}\left(\kappa_{s}, \boldsymbol{x}\right), k=1,3$. These functions solve the Helmholtz equation for the wavenumber $\kappa_{p}$ and the Maxwell's equations for the wavenumber $\kappa_{s}$ [53, Theorems 9.14 and 9.16], respectively.

An incident plane wave defined for $\boldsymbol{x} \in \mathbb{R}^{3}$ by

$$
\boldsymbol{u}^{\text {inc }}(\boldsymbol{x})=\frac{1}{\mu} e^{i \kappa_{s} \boldsymbol{x} \cdot \boldsymbol{d}}(\boldsymbol{d} \times \boldsymbol{p}) \times \boldsymbol{d}+\frac{1}{\lambda+2 \mu} e^{i \kappa_{p} \boldsymbol{x} \cdot \boldsymbol{d}}(\boldsymbol{d} \cdot \boldsymbol{p}) \boldsymbol{d}, \quad \text { where } \boldsymbol{d} \in \mathbf{S}^{2} \text { and } \boldsymbol{p} \in \mathbb{R}^{3},
$$

admits the following series expansion (using [53. Equations (9.44), (9.45), (9.63)] and [28, Theorem 6.26])

$$
\boldsymbol{u}^{\mathrm{inc}}(\boldsymbol{x})=\sum_{m=1}^{\infty} \sum_{j=-m}^{m}\left(\alpha_{m}^{(1)} M_{m, j}^{(1)}\left(\kappa_{s}, \boldsymbol{x}\right)+\beta_{m}^{(1)} N_{m, j}^{(1)}\left(\kappa_{s}, \boldsymbol{x}\right)\right)+\sum_{m=0}^{\infty} \sum_{j=-m}^{m} \gamma_{m}^{(1)} \nabla u_{m, j}^{(1)}\left(\kappa_{p}, \boldsymbol{x}\right),
$$


with the coefficients $\alpha_{m}^{(1)}=\frac{1}{\mu} \frac{4 \pi i^{m}}{m(m+1)} \operatorname{curl}_{\mathbf{S}^{2}} \overline{Y_{m, j}(\boldsymbol{d})} \cdot \boldsymbol{p}, \beta_{m}^{(1)}=\frac{1}{\mu} \frac{4 \pi i^{m}}{m(m+1)} \boldsymbol{\nabla}_{\mathbf{S}^{2}} \overline{Y_{m, j}(\boldsymbol{d})} \cdot \boldsymbol{p}$ and $\gamma_{m}^{(1)}=-\frac{4 \pi i^{m+1}}{\kappa_{p}(\lambda+2 \mu)} \overline{Y_{m, j}(\boldsymbol{d})} \boldsymbol{d} \cdot \boldsymbol{p}$.

Analytical scattered field and far-field pattern. Since the vector spherical harmonics form a complete orthonormal system in the Hilbert space $\boldsymbol{L}^{2}\left(\mathbf{S}^{2}\right)$, the scattered wave and the corresponding far field pattern are given by

$$
\begin{gathered}
\boldsymbol{u}(\boldsymbol{x})=\sum_{m=1}^{\infty} \sum_{j=-m}^{m}\left(\alpha_{m}^{(3)} M_{m, j}^{(3)}\left(\kappa_{s}, \boldsymbol{x}\right)+\beta_{m}^{(3)} N_{m, j}^{(3)}\left(\kappa_{s}, \boldsymbol{x}\right)\right)+\sum_{m=0}^{\infty} \sum_{j=-m}^{m} \gamma_{m}^{(3)} \nabla u_{m, j}^{(3)}\left(\kappa_{p}, \boldsymbol{x}\right), \\
\boldsymbol{u}^{\infty}(\hat{\boldsymbol{x}})=\sum_{m=1}^{\infty} \frac{(-i)^{m+1}}{\kappa_{s}} \sum_{j=-m}^{m}\left(\alpha_{m}^{(3)} \operatorname{curl}_{\mathbf{S}^{2}} Y_{m, j}(\hat{\boldsymbol{x}})+\beta_{m}^{(3)} \boldsymbol{\nabla}_{\mathbf{S}^{2}} Y_{m, j}(\hat{\boldsymbol{x}})\right)+\sum_{m=0}^{\infty}(-i)^{m} \sum_{j=-m}^{m} \gamma_{m}^{(3)} \hat{\boldsymbol{x}} Y_{m, j}(\hat{\boldsymbol{x}})
\end{gathered}
$$

with $\gamma_{0}^{(3)}=-\frac{j_{0}^{\prime}\left(\kappa_{p} R\right)}{h_{0}^{(1)^{\prime}}\left(\kappa_{p} R\right)} \gamma_{0}^{(1)}$ and for $m \geq 1$,

$$
\left(\begin{array}{c}
\alpha_{m}^{(3)} \\
\beta_{m}^{(3)} \\
\gamma_{m}^{(3)}
\end{array}\right)=\left(\begin{array}{ccc}
a_{m}^{(3)} & 0 & 0 \\
0 & b_{m}^{(3,1)} & c_{m}^{(3,1)} \\
0 & b_{m}^{(3,2)} & c_{m}^{(3,2)}
\end{array}\right)^{-1}\left(-\mathbf{U}_{m}^{\text {inc }}\right), \quad \mathbf{U}_{m}^{\text {inc }}=\left(\begin{array}{ccc}
a_{m}^{(1)} & 0 & 0 \\
0 & b_{m}^{(1,1)} & c_{m}^{(1,1)} \\
0 & b_{m}^{(1,2)} & c_{m}^{(1,2)}
\end{array}\right)\left(\begin{array}{c}
\alpha_{m}^{(1)} \\
\beta_{m}^{(1)} \\
\gamma_{m}^{(1)}
\end{array}\right)
$$

where, for $k=1,3$, the coefficients are given by $a_{m}^{(k)}=z_{m}^{(k)}\left(\kappa_{s} R\right), b_{m}^{(k, 1)}=\frac{1}{i \kappa_{s} R}\left(z_{m}^{(k)}\left(\kappa_{s} R\right)+\right.$ $\left.\kappa_{s} R z_{m}^{(k)^{\prime}}\left(\kappa_{s} R\right)\right), c_{m}^{(k, 1)}=\frac{1}{R} z_{m}^{(k)}\left(\kappa_{p} R\right), b_{m}^{(k, 2)}=\frac{m(m+1)}{i \kappa_{s} R} z_{m}^{(k)}\left(\kappa_{s} R\right)$ and $c_{m}^{(k, 2)}=\kappa_{p} z_{m}^{(k)^{\prime}}\left(\kappa_{p} R\right)$. To compute the traction trace of $\boldsymbol{u}$

$$
\boldsymbol{t}_{\mid \Gamma}(\boldsymbol{x})=\sum_{m=1}^{\infty} \sum_{j=-m}^{m}\left(\alpha_{m}^{\mathrm{ex}} \operatorname{curl}_{\mathbf{S}^{2}} Y_{m, j}(\hat{\boldsymbol{x}})+\beta_{m}^{\mathrm{ex}} \nabla_{\mathbf{S}^{2}} Y_{m, j}(\hat{\boldsymbol{x}})\right)+\sum_{m=0}^{\infty} \sum_{j=-m}^{m} \gamma_{m}^{\mathrm{ex}} \hat{\boldsymbol{x}} Y_{m, j}(\hat{\boldsymbol{x}})
$$

we use the following formulas for $k=1,3$ :

$$
\begin{aligned}
\boldsymbol{T}_{\boldsymbol{x}} M_{m, j}^{(k)}(\kappa, \boldsymbol{x})= & \mu\left(\kappa z_{m}^{(k)^{\prime}}(\kappa|\boldsymbol{x}|)-\frac{z_{m}^{(k)}(\kappa|\boldsymbol{x}|)}{|\boldsymbol{x}|}\right) \operatorname{curl}_{\mathbf{S}^{2}} Y_{m, j}(\hat{\boldsymbol{x}}), \\
\boldsymbol{T}_{\boldsymbol{x}} N_{m, j}^{(k)}(\kappa, \boldsymbol{x})= & \frac{\mu}{i \kappa|\boldsymbol{x}|^{2}}\left(-\left(\kappa^{2}|\boldsymbol{x}|^{2}-2 m(m+1)+2\right) z_{m}^{(k)}(\kappa|\boldsymbol{x}|)-2 \kappa|\boldsymbol{x}| z_{m}^{(k)^{\prime}}(\kappa|\boldsymbol{x}|)\right) \boldsymbol{\nabla}_{\mathbf{S}^{2}} Y_{m, j}(\hat{\boldsymbol{x}}) \\
& +2 \mu \frac{m(m+1)}{i \kappa|\boldsymbol{x}|}\left(\kappa z_{m}^{(k)^{\prime}}(\kappa|\boldsymbol{x}|)-\frac{z_{m}^{(k)}(\kappa|\boldsymbol{x}|)}{|\boldsymbol{x}|}\right) \hat{\boldsymbol{x}} Y_{m, j}(\hat{\boldsymbol{x}}) \\
\boldsymbol{T}_{\boldsymbol{x}} \boldsymbol{\nabla} u_{m, j}^{(k)}(\kappa, \boldsymbol{x})= & \frac{2 \mu}{|\boldsymbol{x}|}\left(\kappa z_{m}^{(k)^{\prime}}(\kappa|\boldsymbol{x}|)-\frac{z_{m}^{(k)}(\kappa|\boldsymbol{x}|)}{|\boldsymbol{x}|}\right) \boldsymbol{\nabla}_{\mathbf{S}^{2} Y_{m, j}(\hat{\boldsymbol{x}})} \\
& +\left[-\frac{2 \mu}{|\boldsymbol{x}|^{2}}\left(\left(\kappa^{2}|\boldsymbol{x}|^{2}-m(m+1)\right) z_{m}^{(k)}(\kappa|\boldsymbol{x}|)+2 \kappa|\boldsymbol{x}| z_{m}^{(k)^{\prime}}(\kappa|\boldsymbol{x}|)\right)-\lambda \kappa^{2} z_{m}^{(k)}(\kappa|\boldsymbol{x}|)\right] \hat{\boldsymbol{x}} Y_{m, j}(\hat{\boldsymbol{x}}) .
\end{aligned}
$$

Analytical OSRC-based solution. By using the plane wave expansion (26), we compute the approximate Neumann-type trace of the displacement field corresponding to the OSRC formulation (24):

$$
\begin{aligned}
\tilde{\boldsymbol{t}}_{\mid \Gamma}(\boldsymbol{x}) & =\sum_{m=1}^{\infty} \sum_{j=-m}^{m}\left(\alpha_{m}^{\mathrm{app}} \operatorname{curl}_{\mathbf{S}^{2}} Y_{m, j}(\hat{\boldsymbol{x}})+\beta_{m}^{\mathrm{app}} \boldsymbol{\nabla}_{\mathbf{S}^{2}} Y_{m, j}(\hat{\boldsymbol{x}})\right)+\sum_{m=0}^{\infty} \sum_{j=-m}^{m} \gamma_{m}^{\mathrm{app}} \hat{\boldsymbol{x}} Y_{m, j}(\hat{\boldsymbol{x}}) \\
& =\sum_{m=1}^{\infty} \sum_{j=-m}^{m}\left(\alpha_{2 \mu, m}^{\mathrm{app}} \operatorname{curl}_{\mathbf{S}^{2}} Y_{m, j}(\hat{\boldsymbol{x}})+\beta_{2 \mu, m}^{\mathrm{app}} \boldsymbol{\nabla}_{\mathbf{S}^{2}} Y_{m, j}(\hat{\boldsymbol{x}})\right)+\sum_{m=0}^{\infty} \sum_{j=-m}^{m} \gamma_{2 \mu, m}^{\mathrm{app}} \hat{\boldsymbol{x}} Y_{m, j}(\hat{\boldsymbol{x}})+2 \mu \mathcal{M} \boldsymbol{u}_{\mid \Gamma}(\boldsymbol{x})
\end{aligned}
$$


with $\gamma_{2 \mu, 0}^{\mathrm{app}}=\frac{i \rho \omega^{2}}{\kappa_{p, \varepsilon}} \kappa_{p} j_{0}^{\prime}\left(\kappa_{p} R\right) \gamma_{0}^{(1)}$ and for $m \geq 1$,

$$
\left(\begin{array}{c}
\alpha_{2 \mu, m}^{\mathrm{app}} \\
\beta_{2 \mu, m}^{\mathrm{app}} \\
\gamma_{2 \mu, m}^{\mathrm{app}}
\end{array}\right)=\left(\begin{array}{ccc}
1 & 0 & 0 \\
0 & 1 & d_{m}^{\mathrm{app}, 1} \\
0 & d_{m}^{\mathrm{app}, 2} & 1
\end{array}\right)^{-1}\left(\begin{array}{ccc}
n_{m}^{\mathrm{app}, 1} & 0 & 0 \\
0 & n_{m}^{\mathrm{app}, 2} & 0 \\
0 & 0 & n_{m}^{\mathrm{app}, 3}
\end{array}\right)\left(-\mathbf{U}_{m}^{i n c}\right)
$$

with the coefficients $n_{m}^{\mathrm{app}, 1}=i \mu\left(\kappa_{s, \varepsilon}^{2}-\frac{m(m+1)}{R^{2}}\right)^{\frac{1}{2}}, n_{m}^{\mathrm{app}, 2}=i \rho \omega^{2}\left(\kappa_{s, \varepsilon}^{2}-\frac{m(m+1)}{R^{2}}\right)^{-\frac{1}{2}}, n_{m}^{\mathrm{app}, 3}=$ $i \rho \omega^{2}\left(\kappa_{p, \varepsilon}^{2}-\frac{m(m+1)}{R^{2}}\right)^{-\frac{1}{2}}, d_{m}^{\mathrm{app}, 1}=-i\left(\kappa_{s, \varepsilon}^{2}-\frac{m(m+1)}{R^{2}}\right)^{-\frac{1}{2}} \frac{1}{R}, d_{m}^{\mathrm{app}, 2}=-i\left(\kappa_{p, \varepsilon}^{2}-\frac{m(m+1)}{R^{2}}\right)^{-\frac{1}{2}} \frac{m(m+1)}{R}$.

We have also

$$
\boldsymbol{M} \boldsymbol{u}_{\mid \Gamma}(\boldsymbol{x})=\sum_{m=1}^{\infty} \sum_{j=-m}^{m}\left(\alpha_{m}^{\mathrm{G}} \operatorname{curl}_{\mathbf{S}^{2}} Y_{m, j}(\hat{\boldsymbol{x}})+\beta_{m}^{\mathrm{G}} \nabla_{\mathbf{S}^{2}} Y_{m, j}(\hat{\boldsymbol{x}})\right)+\sum_{m=0}^{\infty} \sum_{j=-m}^{m} \gamma_{m}^{\mathrm{G}} \hat{\boldsymbol{x}} Y_{m, j}(\hat{\boldsymbol{x}})
$$

with $\gamma_{0}^{\mathrm{G}}=-\frac{2}{R} \kappa_{p} j_{0}^{\prime}\left(\kappa_{p} R\right) \gamma_{0}^{1}$ and for $m \geq 1$,

$$
\left(\begin{array}{c}
\alpha_{m}^{\mathrm{G}} \\
\beta_{m}^{\mathrm{G}} \\
\gamma_{m}^{\mathrm{G}}
\end{array}\right)=\left(\begin{array}{ccc}
-\frac{1}{R} & 0 & 0 \\
0 & -\frac{1}{R} & \frac{1}{R} \\
0 & \frac{m(m+1)}{R} & -\frac{2}{R}
\end{array}\right)\left(-\mathbf{U}_{m}^{\mathrm{inc}}\right)
$$

The far-field pattern of the approximate displacement field computed using the OSRC formulation is given by the formula 100 where the far-field operators $\mathscr{F}_{\mathrm{S}}$ and $\mathscr{F}_{\mathrm{D}}$ are defined by (11) and 12 . We use the following analytical formulas

$$
\begin{aligned}
& \mathscr{F}_{\mathbf{S}} \boldsymbol{\nabla}_{\mathbf{S}^{2}} Y_{m, j}(\hat{\boldsymbol{x}})=(-i)^{m-1} \frac{R^{2}}{\mu \kappa_{s}}\left(j_{m}\left(\kappa_{s} R\right)+\kappa_{s} R j_{m}^{\prime}\left(\kappa_{s} R\right)\right) \boldsymbol{\nabla}_{\mathbf{S}^{2}} Y_{m, j}(\hat{\boldsymbol{x}})+(-i)^{m-1} \frac{m(m+1) R}{\kappa_{p}(\lambda+2 \mu)} j_{m}\left(\kappa_{p} R\right) \hat{\boldsymbol{x}} Y_{m, j}(\hat{\boldsymbol{x}} \\
& \mathscr{F}_{\mathbf{S}} \operatorname{curl}_{\mathbf{S}^{2}} Y_{m, j}(\hat{\boldsymbol{x}})=(-i)^{m} \frac{R^{2}}{\mu} j_{m}\left(\kappa_{s} R\right) \operatorname{curl}_{\mathbf{S}^{2}} Y_{m, j}(\hat{\boldsymbol{x}}), \\
& \mathscr{F}_{\mathrm{S}} \hat{\boldsymbol{x}} Y_{m, j}(\hat{\boldsymbol{x}}) \quad=(-i)^{m-1} \frac{R^{2}}{\lambda+2 \mu} j_{m}^{\prime}\left(\kappa_{p} R\right) \hat{\boldsymbol{x}} Y_{m, j}(\hat{\boldsymbol{x}})+(-i)^{m-1} \frac{R}{\mu \kappa_{s}} j_{m}\left(\kappa_{s} R\right) \boldsymbol{\nabla}_{\mathbf{S}^{2}} Y_{m, j}(\hat{\boldsymbol{x}}), \\
& \mathscr{F}_{\mathrm{D}} \boldsymbol{\nabla}_{\mathbf{S}^{2}} Y_{m, j}(\hat{\boldsymbol{x}}) \quad=\frac{(-i)^{m+1}}{\kappa_{s}}\left(\left(\kappa_{s}^{2} R^{2}-2 m(m+1)+2\right) j_{m}\left(\kappa_{s} R\right)+2 \kappa_{s} R j_{m}^{\prime}\left(\kappa_{s} R\right)\right) \boldsymbol{\nabla}_{\mathbf{S}^{2}} Y_{m, j}(\hat{\boldsymbol{x}})+ \\
& \frac{2 \mu(-i)^{m-1}}{(\lambda+2 \mu) \kappa_{p}} m(m+1)\left(\kappa_{p} R j_{m}^{\prime}\left(\kappa_{p} R\right)-j_{m}\left(\kappa_{p} R\right)\right) \hat{\boldsymbol{x}} Y_{m, j}(\hat{\boldsymbol{x}}) \\
& \mathscr{F}_{\mathrm{D}} \operatorname{curl}_{\mathbf{S}^{2}} Y_{m, j}(\hat{\boldsymbol{x}})=(-i)^{m}\left(\kappa_{s} R^{2} j_{m}^{\prime}\left(\kappa_{s} R\right)-R j_{m}\left(\kappa_{s} R\right)\right) \operatorname{curl}_{\mathbf{S}^{2}} Y_{m, j}(\hat{\boldsymbol{x}}) \\
& \mathscr{F}_{\mathrm{D}} \hat{\boldsymbol{x}} Y_{m, j}(\hat{\boldsymbol{x}}) \quad=\frac{(-i)^{m+1}}{\kappa_{p}(\lambda+2 \mu)}\left[2 \mu\left(\left(\kappa_{p}^{2} R^{2}-m(m+1)\right) j_{m}\left(\kappa_{p} R\right)+2 \kappa_{p} R j_{m}^{\prime}\left(\kappa_{p} R\right)\right)+\lambda \kappa_{p}^{2} R^{2} j_{m}\left(\kappa_{p} R\right)\right] \hat{\boldsymbol{x}} Y_{m, j}(\hat{\boldsymbol{x}} \\
& +(-i)^{m-1} \frac{2}{\kappa_{s}}\left(\kappa_{s} R j_{m}^{\prime}\left(\kappa_{s} R\right)-j_{m}\left(\kappa_{s} R\right)\right) \nabla_{\mathbf{S}^{2}} Y_{m, j}(\hat{\boldsymbol{x}}) .
\end{aligned}
$$

[1] F. Alouges, S. Borel, And D. P. Levadoux, A stable well-conditioned integral equation for electromagnetism scattering, J. Comput. Appl. Math., 204 (2007), pp. 440-451.

[2] C. Alves And T. Ha Duong, On the far field amplitude for elastic waves, Modern Mathematical Methods in Diffractions Theory and its Applications in Engineering, (1997), pp. 49-67.

[3] X. Antoine, Fast approximate computation of a time-harmonic scattered field using the osrc method, IMA J. Appl. Math., 66 (2001), pp. 83-110.

[4] X. Antoine, H. Barucq, And A. Bendali, Bayliss-turkel-like radiation condition on surfaces of arbitrary shape, J. Math. Anal. Appl., 229 (1999), pp. 184-211. 
[5] X. Antoine And M. Darbas, Alternative integral equations for the iterative solution of acoustic scattering problems, Quart. J. Mech. Appl. Math., 58 (2005), pp. 107-128.

[6] - Generalized combined field integral equations for the iterative solution of the three-dimensional helmholtz equation, ESAIM: Math. Modell. Numer. Anal., 41 (2007), pp. 147-167.

[7] X. Antoine, M. Darbas, and Y. Y. Lu, An improved surface radiation condition for high-frequency acoustic scattering problems, Comput. Methods Appl. Mech. Engrg., 195 (2006), pp. 4060-4074.

[8] G. A. Baker and P. R. Graves-Morris, Padé approximants, Encyclopedia of Mathematics and its Applications, Cambridge University Press, UK, 1996.

[9] H. Barucq, R. Djellouli, And A.-G. Saint-Guirons, Performance assessment of a new class of local absorbing boundary conditions for elliptical- and prolate spheroidal-shaped boundaries, Applied Numerical Mathematics, 59 (2009), pp. 1467-1498.

[10] H. Barucq, R. Djellouli, And A.-G. Saint-Guirons, High-frequency analysis of the efficiency of a local approximate DtN2 boundary condition for prolate spheroidal-shaped boundaries., Wave motion, 47 (2010), pp. 583-600.

[11] A. Bayliss And E. Turkel, Radiation boundary conditions for wave-like equations., Comm. Pure Appl. Math., 33 (1980), pp. 707-725.

[12] J.-P. Berenger, A perfectly matched layer for the absorption of electromagnetic waves, Journal of computational physics, 114 (1994), pp. 185-200.

[13] J. Bielak, K. Loukakis, Y. Hisada, and C. Yoshimura, Domain reduction method for threedimensional earthquake modeling in localized regions, part $i$ : Theory, Bulletin of the Seismological Society of America, 93 (2003), pp. 817-824.

[14] Y. Boubendir, X. Antoine, and C. Geuzaine, A quasi-optimal non-overlapping domain decomposition algorithm for the helmholtz equation, Journal of Computational Physics, 231 (2012), pp. 262-280.

[15] Y. Boubendir And C. Turc, Well-conditioned boundary integral equation formulations for the solution of high-frequency electromagnetic scattering problems, Computers and Mathematics with Applications, 67 (2014), pp. 1772-1805.

[16] O. Bruno, T. Elling, And C. Turc, Regularized integral equations and fast high-order solvers for sound-hard acoustic scattering problems, Int. Journal Numerical Methods in Engineering, 91 (2012), pp. 1045-1072.

[17] B. CARPEntieri, A matrix-free two-grid preconditioner for solving boundary integral equations in electromagnetism, Computing, 77 (2006), pp. 275-296.

[18] B. Carpentieri, I. Duff, and L. Giraud, Some sparse pattern selection strategies for robust frobenius norm minimization preconditioners in electromagnetism, Numer. Linear Algebra Appl., 7 (2000), pp. 667-685.

[19] B. Carpentieri, I. S. Duff, L. Giraud, And G. Sylvand, Combining fast multipole techniques and an approximate inverse preconditioner for large parallel electromagnetics calculations, SIAM J. Sci. Comp., 27 (2005), pp. 774-792.

[20] C. Cerjan, D. Kosloff, R. Kosloff, and M. Reshef, A nonreflecting boundary condition for discrete acoustic and elastic wave equations, Geophysics, 50 (1985), pp. 705-708.

[21] S. Chaillat And M. Bonnet, Recent advances on the fast multipole accelerated boundary element method for 3D time-harmonic elastodynamics, Wave Motion, 50 (2013), pp. 1090-1104.

[22] S. Chaillat, M. Bonnet, and J. F. Semblat, A multi-level fast multipole BEM for 3-D elastodynamics in the frequency domain, Computer Methods in Applied Mechanics and Engineering, 197 (2008), pp. 4233-4249. 
[23] —_, A new fast multi-domain BEM to model seismic wave propagation and amplification in $3 D$ geological structures, Geophys. J. Int., 177 (2009), pp. 509-531.

[24] S. Chaillat, J. F. Semblat, And M. Bonnet, A preconditioned 3-d multi-region fast multipole solver for seismic wave propagation in complex geometries., Commun. Comp. Phys., 11 (2012), pp. 594-609.

[25] E. Chaljub, D. Komatitsch, J.-P. Vilotte, Y. Capdeville, B. Valette, and G. Festa, Spectral-element analysis in seismology, Advances in Geophysics, 48 (2007), pp. 365-419.

[26] W. Chew And Q. Liu, Perfectly matched layers for elastodynamics: a new absorbing boundary condition, Journal of Computational Acoustics, 4 (1996), pp. 341-359.

[27] R. Clayton And B. Engquist, Absorbing boundary conditions for acoustic and elastic wave equations, Bulletin of the Seismological Society of America, 67 (1977), pp. 1529-1540.

[28] D. L. Colton And R. Kress, Inverse acoustic and electromagnetic scattering theory, vol. 93 of Applied Mathematical Sciences, Springer-Verlag, Berlin, second ed., 1998.

[29] M. Darbas, Préconditionneurs analytiques de type Calderón pour les formulations intégrales des problèmes de diffraction d'ondes, PhD Thesis, INSA, Toulouse, 2004.

[30] M. Darbas, Generalized combined field integral equations for the iterative solution of the threedimensional Maxwell equations, Appl. Math. Lett., 19 (2006), pp. 834-839.

[31] M. Darbas, E. Darrigrand, And Y. Lafranche, Combining OSRC preconditioning and Fast Multipole Method for the Helmholtz equation, J. Comput. Phys., 236 (2013), pp. 289-316.

[32] M. Darbas And F. LE LouËr, Well-conditioned boundary integral formulations for the iterative solution of elastic scattering problems, Mathematical Methods in the Applied Sciences, 38 (2015), pp. 1705-1733.

[33] M. El Bouajaji, X. Antoine, and C. Geuzaine, Approximate local magnetic-to-electric surface operators for time-harmonic maxwell's equations, Journal of Computational Physics, 279 (2014), pp. 241-260.

[34] B. Engquist And A. Majda, Radiation boundary conditions for acoustic and elastic wave calculations, Comm. Pure Appl. Math., 32 (1979), pp. 314-358.

[35] G. K. GÄChter And M. J. Grote, Dirichlet-to-Neumann map for three-dimensional elastic waves, Wave Motion, 37 (2003), pp. 293-311.

[36] M. Gander, F. Magoules, and F. Nataf, Optimized Schwarz methods without overlap for the Helmholtz equation, SIAM J. Sci. Comput., 24 (2002), pp. 38-60.

[37] D. Givoli, High-order nonreflecting boundary conditions without high-order derivatives, Journal of Computational Physics, 122 (2001), pp. 849-870.

[38] R. W. Graves, Simulating seismic wave propagation in 3d elastic media using staggered-grid finite differences, Bulletin of the Seismological Society of America, 86 (1996), pp. 1091-1106.

[39] M. Grote and J. Keller, Exact nonreflecting boundary condition for elastic waves, SIAM J. Appl. Math., 60 (2000), pp. 803-818.

[40] L. Halpern, S. Petit-Bergez, and J. Rauch, The analysis of matched layers, Confluentes Mathematici, 3 (2011), pp. 159-236.

[41] J. S. Hesthaven And T. Warburton, Nodal discontinuous Galerkin methods: algorithms, analysis, and applications, vol. 54, Springer, 2007.

[42] D. S. Jones, An approximate boundary condition in acoustics, J. Sound Vibr., 121 (1998), pp. 37-45.

[43] D. Komatitsch And J.-P. Vilotte, The spectral element method: an efficient tool to simulate the seismic response of $2 d$ and $3 d$ geological structures, Bulletin of the seismological society of America, 88 (1998), pp. 368-392. 
[44] G. A. Kriegsmann, A. Taflove, and K. R. Umashankar, A new formulation of electromagnetic wave scattering using the on-surface radiation condition method, IEEE Trans. Antennas Propag., 35 (1987), pp. 153-161.

[45] V. D. Kupradze, T. G. Gegelia, M. O. Basheleřshvili, and T. V. Burchuladze, Threedimensional problems of the mathematical theory of elasticity and thermoelasticity, vol. 25 of NorthHolland Series in Applied Mathematics and Mechanics, North-Holland Publishing Co., Amsterdam, russian ed., 1979. Edited by V. D. Kupradze.

[46] F. LE LOUËR, A high order spectral algorithm for elastic obstacle scattering in three dimensions, J. Comput. Phys., 279 (2014), pp. 1-17.

[47] Y. J. Liu, S. Mukherjee, N. Nishimura, M. Schanz, W. Ye, A. Sutradhar, E. Pan, N. A. Dumont, A. Frangi, And A. Saez, Recent Advances and Emerging Applications of the Boundary Element Method, Appl. Mech. Rev., 64 (2011), p. 030802.

[48] Y. Y. Lu, A complex rational approximation of $\sqrt{1+x}$, Applied Numerical Mathematics, 27 (1998), pp. 141-154.

[49] F. Magoules, F.-X. Roux, and S. Salmon, Optimal discrete transmission conditions for a nonoverlapping domain decomposition method for the helmholtz equation, SIAM Journal on Scientific Computing, 25 (2004), pp. 1497-1515.

[50] F. Magoules, F.-X. Roux, And L. Series, Algebraic approximation of dirichlet-to-neumann maps for the equations of linear elasticity, Comput. Methods Appl. Mech. Engrg., 195 (2006), pp. 37423759 .

[51] M. Medvinsky And E. Turkel, On surface radiation conditions for an ellipse, J. Comput. Appl. Math., 234 (2010), pp. 1647-1655.

[52] F. A. Milinazzo, C. A. Zala, And G. H. Brooke, Rational square-root approximations for parabolic equation algorithms, J. Acoust. Soc. Am., 101 (1997), pp. 760-766.

[53] P. Monk, Finite element methods for Maxwell's equations, Numerical Mathematics and Scientific Computation, Oxford University Press, New York, 2003.

[54] J.-C. NÉDÉLEC, Acoustic and electromagnetic equations, vol. 144 of Applied Mathematical Sciences, Springer-Verlag, New York, 2001. Integral representations for harmonic problems.

[55] E. H. Saenger, N. Gold, and S. A. Shapiro, Modeling the propagation of elastic waves using a modified finite-difference grid, Wave motion, 31 (2000), pp. 77-92.

[56] J. Virieux, H. Calandra, and R.-É. Plessix, A review of the spectral, pseudo-spectral, finitedifference and finite-element modelling techniques for geophysical imaging, Geophysical Prospecting, 59 (2011), pp. 794-813. 